Pontifícia Universidade Católica $_{\text {a }}$

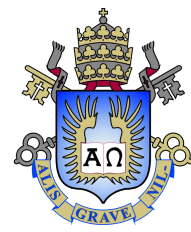

Simone de Freitas de Souza

\title{
A construção de Delzant para variedades tóricas simpléticas
}

Dissertação de Mestrado

Dissertação apresentada como requisito parcial para obtenção do grau de Mestre pelo Programa de Pós-Graduação em Matemática do Departamento de Matemática do Centro Técnico Científico da PUC-Rio.

Orientadora: Prof. Alessia Mandini 


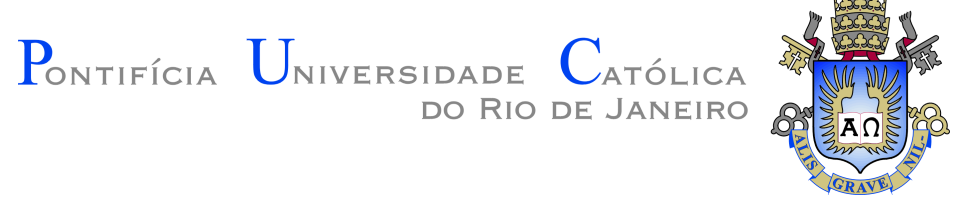

Simone de Freitas de Souza

\section{A construção de Delzant para variedades tóricas simpléticas}

Dissertação apresentada como requisito parcial para obtenção do grau de Mestre pelo Programa de Pós-Graduação em Matemática do Departamento de Matemática do Centro Técnico Científico da PUC-Rio. Aprovada pela Comissão Examinadora abaixo assinada.

Prof. Alessia Mandini

Orientadora

Departamento de Matemática - PUC-Rio

Prof. David Martínez Torres

Departamento de Matemática - PUC-Rio

Prof. Marcos Craizer

Departamento de Matemática - PUC-Rio

Prof. Marta Batoréo

Universidade Federal do Espírito Santo - UFES

Prof. Márcio da Silveira Carvalho

Coordenador Setorial do Centro Técnico Científico - PUC-Rio

Rio de Janeiro, 27 de Setembro de 2018 
Todos os direitos reservados. É proibida a reprodução total ou parcial do trabalho sem autorização da universidade, do autor e do orientador.

\section{Simone de Freitas de Souza}

Especialista Lato Sensu pelo curso de Pós-Graduação da Universidade Federal Fluminense em Novas Tecnologias no Ensino da Matemática, em 2016. Terminou sua graduação em Matemática na Universidade Federal Fluminense no ano de 2011. Atualmente, exerce o cargo Professor I no Colégio Estadual Paulo Assis Ribeiro. Possui experiência na área de licenciatura em Matemática.

Ficha Catalográfica

De Souza, Simone de Freitas

A construção de Delzant para variedades tóricas simpléticas / Simone de Freitas de Souza; orientador: Alessia Mandini. $-2018$.

v., 80 f: il. color. ; $30 \mathrm{~cm}$

Dissertação (mestrado) - Pontifícia Universidade Católica do Rio de Janeiro, Departamento de Matemática.

Inclui bibliografia

1. Variedades Tóricas Simpléticas;. 2. Construção de Delzant;. 3. Aplicação Momento;. 4. Polítopo de Delzant;. 5. Teorema de Convexidade..

I. Mandini, Alessia. II. Pontifícia Universidade Católica do Rio de Janeiro. Departamento de Matemática. III. Título. 
Dedico, In Memoriam, ao meu amado pai Cláudio José de Souza. 


\section{Agradecimentos}

A Deus, pela oportunidade que me concedeu, por providenciar todo o necessário, por sempre me capacitar e colocar pessoas especiais no meu caminho, citadas neste momento, que colaboraram para tornar a minha caminhada mais fácil.

A minha mãe Nilsea, minha irmã Cláudia e ao meu namorado Tadeu, que souberam compreender esse período em que não pude estar sempre presente, por serem meu sustento, meu porto seguro em todos os momentos e por acreditarem em mim.

Aos meus amigos da PUC-Rio, sempre dispostos a ajudar, seja na compreensão de alguns conteúdos, seja como ombro amigo ou alegrando em diversos encontros. Destaco aqui o Edison, Thiago e João. Também o Rodrigo, por ter antecipado meus planos de mestrado por enxergar em mim um potencial que eu mesma não via.

A minha orientadora Alessia Mandini pela sugestão do tema, o qual me cativou desde o início, pelo direcionamento primordial na preparação, pela disposição e paciência.

Ao Raimundo Neto Nunes Leão pelas explicações, auxílios e principalmente pelos conselhos essenciais dados a mim.

Aos professores e toda equipe do departamento de matemática da PUCRio, por tamanha dedicação em seu trabalho, em particular a Creuza Nascimento.

Ao professor Francisco Fontenele Neto, que intensificou meu interesse e admiração pela geometria no período em que estudei na UFF.

O presente trabalho foi realizado com apoio da Coordenação de Aperfeiçoamento de Pessoal de Nível Superior - Brasil (CAPES) - Código de Financiamento 001. 


\section{Resumo}

De Souza, Simone de Freitas; Mandini, Alessia. A construção de Delzant para variedades tóricas simpléticas. Rio de Janeiro, 2018. 80p. Dissertação de Mestrado - Departamento de Matemática, Pontifícia Universidade Católica do Rio de Janeiro.

Em 1988, Delzant classificou as variedades compactas tóricas simpléticas por meio da imagem associada da aplicação momento. Como estabelecido pelo Teorema de Convexidade [Atiyah, Guillemin-Sternberg, 1983], a imagem pela aplicação momento de uma variedade compacta tórica simplética é um polítopo convexo. A construção de Delzant proporciona uma receita para formar, dado um polítopo de Delzant, uma variedade compacta tórica simplética. Nesta dissertação revisamos essa construção e estudamos alguns exemplos.

\section{Palavras-chave}

Variedades Tóricas Simpléticas; Construção de Delzant; Aplicação Momento; Polítopo de Delzant; Teorema de Convexidade. 


\section{Abstract}

De Souza, Simone de Freitas; Mandini, Alessia (Advisor). Delzant's construction for toric symplectic manifolds. Rio de Janeiro, 2018. 80p. Dissertação de Mestrado - Departamento de Matemática, Pontifícia Universidade Católica do Rio de Janeiro.

In 1988, Delzant proved a classification Theorem of compact toric symplectic manifolds by means of their moment image. By the convexity Theorem [Atiyah, Guillemin-Sternberg, 1983] the moment image of a compact toric symplectic manifold is a convex polytope. Delzant's construction gives a recipe to construct, given a Delzant polytope, the corresponding compact toric symplectic manifold. This thesis describes this construction and studies in detail some examples.

\section{Keywords}

Toric Symplectic Manifolds; Delzant's Construction; Moment Map; Delzant Polytope; Convexity Theorem. 


\section{Sumário}

1 Introdução 10

2 Variedades Simpléticas $\quad 12$

2.1 Conceitos Iniciais 12

2.2 Campo Vetorial Hamiltoniano 15

3 Sistemas Integráveis $\quad 22$

4 Ações de Grupos Sobre Variedades $\quad 29$

4.1 Ações de grupos $\quad 29$

4.2 Ações Hamiltonianas 30

4.3 Ações Tóricas Hamiltonianas 33

5 Teorema de Delzant $\quad 44$

6 Redução Simplética $\quad 49$

6.1 Espaço das Órbitas 49

6.2 O Teorema de Marsden-Weinstein-Meyer 51

$\begin{array}{lll}7 & \text { Construção de Delzant } & 57\end{array}$

8 Exemplos da Construção de Delzant $\quad 63$

8.1 Segmento de Comprimento $L \quad 63$

8.2 Triângulo Retângulo de Catetos $L$

8.3 Quadrado de Lado $L \quad 68$

8.4 Trapézio 71

$\begin{array}{lll}8.5 & \text { Tetraedro } & 75\end{array}$

$\begin{array}{lll}\text { 8.6 Cubo de Lado } L & 77\end{array}$

$\begin{array}{ll}\text { Referências bibliográficas } & 79\end{array}$ 


\section{Lista de figuras}

5.1 1-polítopo de Delzant 45

5.2 1-polítopo não Delzant 45

5.3 2-polítopo de Delzant 45

5.4 2-polítopo não Delzant 46

5.5 3-polítopo de Delzant 46

5.6 3-polítopo não Delzant 46

5.7 Vetores normais primitivos 48 


\section{Introdução}

Esse trabalho se baseia no estudo das variedades tóricas simpléticas $2 n$ dimensionais, mais especificamente, a associação de cada uma delas a um objeto no $\mathbb{R}^{n}$, chamado polítopo, através de uma correspondência dada pela imagem da aplicação momento. Tal fato é afirmado no Teorema de Delzant, que estabelece que essa correspondência é biunívoca, provado no artigo original [1].

A ferramenta crucial para a construção da variedade, dado o seu polítopo de Delzant, é o quociente simplético por meio do Teorema de redução simplética de Marsden-Weinstein-Meyer ([2], [3]). Para isso, estudaremos variedades simpléticas munidas da ação de um grupo de Lie que preserva a estrutura simplética, mais especificamente, ações simpléticas que possuem uma aplicação momento, conhecidas como ações hamiltonianas.

Começamos, no capítulo 2, introduzindo conceitos iniciais tais como variedade simplética e campos vetoriais hamiltonianos, além de enunciar o Teorema de Darboux, que mostra que toda variedade simplética possui um atlas simplético, cujas mudanças de cartas preservam a forma simplética.

No terceiro capítulo iremos relembrar a noção de álgebra de Lie, fundamental nas ações hamiltonianas e sistemas integráveis. Será enunciado o Teorema de Arnold-Liouville.

No capítulo 4 estudamos as ações de grupos sobre variedades, mais precisamente as ações hamiltonianas: de um grupo de Lie sobre uma variedade simplética e, em particular, de um toro $n$-dimensional sobre uma variedade simplética $2 n$-dimensional (ações tóricas). Será definida a variedade tórica simplética e enunciado o teorema de Convexidade.

O capítulo 5 defimos o polítopo de Delzant, será enunciado o teorema central deste trabalho, o Teorema de Delzant, que associa biunivocamente a um polítopo de Delzant (a menos de equivalência pela ação do grupo afim $\operatorname{Aff}_{\mathbb{Z}}\left(\mathbb{R}^{n}\right)$ ) uma variedade tórica simplética (a menos de simplectomorfismos) através da imagem da aplicação momento.

No capítulo 6 vamos descrever a redução simplética, técnica que diminui o total de simetrias por meio da ação hamiltoniana de um grupo e sua aplicação momento. Como elemento final, teremos uma variedade simplética dada pelo 
par quociente simplético e forma reduzida.

Nos dois últimos capítulos encerramos com a construção de Delzant, parte da teoria descrita em seu artigo que, mais informalmente, se traduz em uma receita para a construção da variedade tórica simplética associada a um polítopo de Delzant conhecido. A conclusão se dará com alguns exemplos dessa construção. 


\section{2}

\section{Variedades Simpléticas}

Neste capítulo iremos definir o conceito de variedade simplética e enunciar o Teorema de Darboux.

\section{1}

\section{Conceitos Iniciais}

Definição 2.1 Seja $M$ uma variedade diferenciável. Uma 2-forma ${ }^{a} \omega \in \Omega^{2}(M)$ é dita simplética se $\omega$ é:

i) fechada: i.e. $d \omega=0$,

ii) não degenerada: i.e. $\forall p \in M$ a forma bilinear $\omega_{p}: T_{p} M \times T_{p} M \rightarrow \mathbb{R}$ em $p$ é não degenerada:

$$
\omega_{p}(X, Y)=0, \forall Y \in T_{p} M \Rightarrow X=0
$$

Definição 2.2 O par $(M, \omega)$, de uma variedade suave e uma 2-forma simplética, é chamado de variedade simplética.

Algumas consequências:

i) anti-simetria: como toda 2-forma é anti-simétrica, vale

$$
\omega_{p}(X, Y)=-\omega_{p}(Y, X), \forall p \in M,
$$

ii) a variedade $M$ possui dimensão par: se $A_{p}$ é a matriz $n \times n$ associada a $\omega_{p}$, então $A_{p}$ é não singular. Logo

$$
A_{p}=-A_{p}^{T}
$$

${ }^{a}$ Para consultar assuntos tais como variedades diferenciáveis, formas diferenciais e operações entre elas recomendamos [4], [5], [6]. 
Assim,

$$
\operatorname{det}\left(A_{p}\right)=\operatorname{det}\left(-A_{p}^{T}\right)=(-1)^{n} \operatorname{det}\left(A_{p}\right) .
$$

Portanto, $n$ é par.

Definição 2.3 Seja $U$ subconjunto aberto de uma variedade $M$. Uma $k$-forma diferencial $\omega \in \Omega^{k}(U)$ é exata se existe uma $(k-1)$-forma $\alpha \in \Omega^{k-1}(U)$ satisfazendo $d \alpha=\omega$. Neste caso, $\alpha$ é chamado de potencial para $\omega$.

Proposição 2.4 Localmente toda forma fechada é exata.

Prova. Considere $M$ variedade diferenciável e $\omega \in \Omega^{k}(M), k \geqslant 1$. Para cada $p \in M$, existe $U_{p}$ vizinhança de $p$ tal que

$$
d \omega=0 \Rightarrow \exists \alpha \in \Omega^{k-1}\left(U_{p}\right) \text { tal que } \omega=d \alpha .
$$

\section{Exemplos}

1. Seja $M=\mathbb{R}^{2 n}$ com coordenadas $x_{1}, \ldots, x_{n}, y_{1}, \ldots, y_{n}$ e considere a 2 -forma

$$
\omega_{0}=\sum_{k=1}^{n} d x_{k} \wedge d y_{k} .
$$

Verifiquemos que a forma $\omega_{0}$ é simplética:

i) a forma $\omega_{0}$ é fechada: porque é exata.

Por exemplo, $\omega_{0}=-d \alpha$ para $\alpha=\sum_{i} y_{i} d x_{i}$.

ii) a forma $\omega_{0}$ é não degenerada: seja $v \in T_{p} M, \omega_{0} \in \Omega^{2}(M)$ tal que

$$
\omega_{0}(u, v)=0, \forall u \in T_{p} M
$$

queremos mostrar que $v=0$. Seja $u=\sum_{j=1}^{n}\left(a_{j} \frac{\partial}{\partial x_{j}}+b_{j} \frac{\partial}{\partial y_{j}}\right)$, então

$$
\begin{aligned}
\omega_{0}(u, v) & =\sum_{k=1}^{n} d x_{k} \wedge d y_{k}\left(\sum_{j=1}^{n}\left(a_{j} \frac{\partial}{\partial x_{j}}+b_{j} \frac{\partial}{\partial y_{j}}\right), v\right) \\
& =\sum_{k=1}^{n} d x_{k} \wedge d y_{k}\left(a_{1} \frac{\partial}{\partial x_{1}}, v\right)+\sum_{k=1}^{n} d x_{k} \wedge d y_{k}\left(b_{1} \frac{\partial}{\partial y_{1}}, v\right)+\cdots+ \\
& +\cdots+\sum_{k=1}^{n} d x_{k} \wedge d y_{k}\left(a_{n} \frac{\partial}{\partial x_{n}}, v\right)+\sum_{k=1}^{n} d x_{k} \wedge d y_{k}\left(b_{n} \frac{\partial}{\partial y_{n}}, v\right) \\
& =a_{1} d y_{1}(v)-b_{1} d x_{1}(v)+\cdots+a_{n} d y_{n}(v)-b_{n} d x_{n}(v) \\
& =\sum_{j=1}^{n}\left(a_{j} d y_{j}(v)-b_{j} d x_{j}(v)\right)=0
\end{aligned}
$$


Da igualdade acima, segue que $v=0$ ou $v=u$. Como $v$ é fixo, descartamos a segunda opção. Portanto, $v=0$. Assim, o par $\left(\mathbb{R}^{2 n}, \sum_{k=1}^{n} d x_{k} \wedge d y_{k}\right)$ é uma variedade simplética.

2. Seja $M=\mathbb{S}^{2}$ a esfera unitária em $\mathbb{R}^{3}$ e $T_{p} \mathbb{S}^{2}$ o espaço vetorial tangente em $p$ a $\mathbb{S}^{2}$. A forma simplética canônica em $\mathbb{S}^{2}$ é a forma induzida pelo produto misto

$$
\omega_{p}(u, v):=\langle p, u \times v\rangle, \forall p \in \mathbb{S}^{2}, u, v \in T_{p} \mathbb{S}^{2}
$$

i) a forma $\omega$ é fechada porque é de grau máximo (forma volume);

ii) a forma $\omega$ é não degenerada: de fato, $\forall p \in \mathbb{S}^{2}, u \in T_{p} \mathbb{S}^{2}, u \neq 0$, existe $v \in T_{p} \mathbb{S}^{2}$ tal que $\langle p, u \times v\rangle \neq 0$. Por exemplo, considere $v=u \times p$. Logo, $\left(\mathbb{S}^{2}, \omega\right)$ é uma variedade simplética.

3. O cilindro $\mathbb{S}^{1} \times \mathbb{R}$ com coordenadas $(\theta, h)$ é uma variedade simplética com a forma canônica $\omega=d \theta \wedge d h$.

i) a forma $\omega$ é fechada porque é exata. Por exemplo, $\omega=d \alpha$ com $\alpha=h d \theta$;

ii) a forma $\omega$ é não degenerada: de fato, $\forall p=(\theta, h) \in \mathbb{S}^{1} \times \mathbb{R}$, os vetores tangentes $u, v \in T_{p}\left(\mathbb{S}^{1} \times \mathbb{R}\right)$ são da forma

$$
u=a_{u} \frac{\partial}{\partial \theta}+b_{u} \frac{\partial}{\partial h}, v=a_{v} \frac{\partial}{\partial \theta}+b_{v} \frac{\partial}{\partial h},
$$

$\operatorname{com} a_{u}, b_{u}, a_{v}, b_{v} \in \mathbb{R}$. Assim,

$$
\omega_{p}(u, v)=d \theta \wedge d h(u, v)=a_{u} b_{v}-a_{v} b_{u}
$$

Logo, para cada vetor $u$ não nulo existe pelo menos um vetor $v$ tal que $\omega_{p}(u, v) \neq 0$ e, consequentemente, $\omega_{p}$ é não degenerada. Assim, como o argumento é verdadeiro para qualquer $p \in \mathbb{S}^{1} \times \mathbb{R}$, temos que $\omega$ é não degenerada. ${ }^{b}$

Definição 2.5 Sejam $\left(M_{1}, \omega_{1}\right)$ e $\left(M_{2}, \omega_{2}\right)$ variedades simpléticas $2 n$ dimensionais. Um simplectomorfismo ${ }^{c}$ entre as duas variedades é um difeomorfismo $\varphi: M_{1} \rightarrow M_{2}$ tal que $\varphi^{*} \omega_{2}=\omega_{1}$. Isto é, se $\varphi$ preserva as formas

${ }^{b}$ Observe que, dada a projeção $\pi: \mathbb{S}^{1} \times \mathbb{R} \longrightarrow \mathbb{S}^{1}$, o quociente herda uma estrutura simplética da original, através das cópias por difeomorfismos. Ou seja, existe uma única estrutura simplética em $\mathbb{S}^{1}$ que torna a projeção $\pi$ uma aplicação de recobrimento.

${ }^{c}$ Recomendamos a leitura de [7]. 
simpléticas. Neste caso, dizemos que $\left(M_{1}, \omega_{1}\right)$ e $\left(M_{2}, \omega_{2}\right)$ são simplectomorfas.

Teorema 2.6 (Darboux) Seja $\left(M^{2 n}, \omega\right)$ uma variedade simplética e $p \in M$. Então, existe uma carta coordenada $\left(U, x_{1}, \ldots, x_{n}, y_{1}, \ldots, y_{n}\right)$ centrada em $p$ tal que

$$
\left.\omega\right|_{U}=\sum_{i=1}^{n} d x_{i} \wedge d y_{i}
$$

tal carta é chamada Carta de Darboux.

Aqui o Teorema de Darboux não será demonstrado, mas faremos alguns comentários. Ele afirma que uma variedade simplética $\left(M^{2 n}, \omega\right)$ é localmente simplectomorfa a um aberto de $\mathbb{R}^{2 n}$, com estrutura simplética $\omega_{0}:=\sum_{i=1}^{n} d q_{i} \wedge d p_{i}$. Ou seja, todo $p \in M^{2 n}$ possui vizinhança simplectomorfa a uma vizinhança de $0 \in \mathbb{R}^{2 n}$ munida da forma simplética canônica. Como consequência, toda variedade simplética possui um atlas simplético (aquele cujas mudanças de cartas preservam a forma simplética). O Teorema de Darboux mostra que formas simpléticas são localmente rígidas, além de fornecer uma classificação local de variedades simpléticas em termos de um único invariante: a dimensão. Para a demonstração, consulte [8].

\section{2}

\section{Campo Vetorial Hamiltoniano}

Seja $(M, \omega)$ uma variedade simplética.

Definição 2.7 Dado um campo vetorial $X \in \mathfrak{X}(M)$ e uma $k$-forma diferencial $\omega \in \Omega^{k}(M)$, a contração ou o produto interior de $\omega$ por $X$ é a $(k-1)$-forma $i_{X} \omega \in \Omega^{k-1}(M)$ definida por

$$
i_{X} \omega\left(X_{1}, \ldots, X_{k-1}\right)=\omega\left(X, X_{1}, \ldots, X_{k-1}\right) .
$$

Dados dois campos vetoriais $X, Y: M \rightarrow T M$, podemos construir uma aplicação $X Y: M \rightarrow T M$ através da igualdade $X Y f=X(Y f), \forall f \in C^{\infty}(M)$. Em geral, $X Y$ não é um campo vetorial em $M$, mas é possível obter um novo campo vetorial a partir de $X$ e $Y$ denotado por $[X, Y]$ através da expressão

$$
[X, Y]=X Y-Y X
$$

chamada de colchete de Lie nos campos $X$ e $Y$.

Observação: $\mathrm{O}$ colchete de Lie entre os dois campos $X, Y \in \mathfrak{X}(M)$ mede a comutatividade entre $X$ e $Y$. Assim, se $X$ e $Y$ comutam, temos $[X, Y]=0$. 
Nesse caso, $X Y: M \rightarrow T M$ determina um campo vetorial em $M$.

Definição 2.8 Para quaisquer campos vetoriais suaves $V$ e $W$ em $M$, a derivada de Lie do campo vetorial $W$ na direção do campo $V$ na variedade $M$ é dada pela expressão

$$
\mathcal{L}_{V} W=[V, W]
$$

Proposição $2.9^{d}$ Sejam $f \in C^{\infty}(M)$, df a 1-forma correspondente e $X$ um campo vetorial em $M$, então

$$
\mathcal{L}_{X} f=d f(X)=X(f) .
$$

Definição 2.10 Um campo vetorial $X$ em $M$ é simplético se a contração $i_{X} \omega$ é fechada $\left(d i_{X} \omega=0\right)$.

Pela Fórmula de Cartan

$$
\mathcal{L}_{X} \omega=d i_{X} \omega+i_{X} d \omega=0
$$

o fluxo de um campo vetorial simplético preserva a forma $\left(\mathcal{L}_{X} \omega=0\right)$.

Definição 2.11 Um campo vetorial $X_{H}$ em $M$ é hamiltoniano se a contração $i_{X_{H}} \omega$ é exata.

Disto, segue que os campos hamiltonianos são simpléticos. Resumindo,

$$
\begin{aligned}
X_{H} \text { campo simplético } & \Leftrightarrow i_{X_{H}} \omega \in \Omega^{1}(M) \text { é fechada } \\
& \Uparrow \\
X_{H} \text { campo hamiltoniano } & \Leftrightarrow i_{X_{H}} \omega \in \Omega^{1}(M) \text { é exata. }
\end{aligned}
$$

Definição 2.12 Um campo vetorial $X_{H}$ em $M$ é localmente hamiltoniano se para cada ponto $p \in M$ existe uma vizinhança $U_{p}$ tal que $X_{H}$ é hamiltoniano em $U_{p}$.

Definição 2.13 Uma função suave $H: M \longrightarrow \mathbb{R}$ é dita função hamiltoniana para o campo vetorial $X$ em $(M, \omega)$ se $i_{X} \omega=d H$.

${ }^{d} \mathrm{~A}$ demonstração se encontra em [9]. 
Proposição 2.14 Se $M$ é variedade simplética, dada a função hamiltoniana $H: M \rightarrow \mathbb{R}, H \in C^{\infty}(M)$ e seu campo vetorial $X_{H} \in \mathfrak{X}(M)$ satisfazendo a equação de Hamilton $i_{X_{H}} \omega=d H$, o campo $X_{H} \in \mathfrak{X}(M)$ é único (neste caso, chamado de campo hamiltoniano ou gradiente simplético da hamiltoniana $H$ ).

Prova. Suponha que existam $X_{1}, X_{2} \in \mathfrak{X}(M)$ satisfazendo $i_{X_{j}} \omega=d H$, para $j=1,2$. Então,

$$
\begin{aligned}
\forall Y \in \mathfrak{X}(M), \quad \omega\left(X_{1}-X_{2}, Y\right) & =\omega\left(X_{1}, Y\right)-\omega\left(X_{2}, Y\right) \\
& =d H(Y)-d H(Y) \\
& =0
\end{aligned}
$$

Como $\omega$ é não degenerada e obtemos esse resultado para um $Y$ qualquer, temos $X_{1}=X_{2}$.

Lema 2.15 $X \in \mathfrak{X}(M)$ é simplético se, e somente se, for localmente hamiltoniano.

Prova. Suponha $X$ simplético. Pela Proposição 2.4, para cada $p \in M$, existe $U_{p}$, vizinhança de $p$, tal que $i_{X} \omega \in \Omega^{1}\left(U_{p}\right)$ é exata. Então, existe $H: U_{p} \rightarrow \mathbb{R}$, para $H \in C^{\infty}\left(U_{p}\right)$ tal que

$$
i_{X} \omega=d H \underbrace{\Rightarrow}_{\omega \text { é nãodegenerada }} X=X_{H} \text { em } U_{p}
$$

ou seja,

$$
i_{X} \omega=d H=i_{X_{H}} \omega
$$

assim, $X$ é hamiltoniano em $U_{p}$. Suponha $X$ localmente hamiltoniano. Então, para cada $p \in M$ existe $U_{p}$ vizinhança de $p$ e $H: U_{p} \rightarrow \mathbb{R}$ diferenciável tal que $d H=\left.i_{X} \omega\right|_{U_{p}}$. Assim,

$$
i_{X} \omega=i_{X_{H}} \omega=d H
$$

Logo, $i_{X} \omega$ é exata e, portanto, fechada. Portanto, $X$ é simplético.

Proposição 2.16 $O$ fluxo de $X_{H}$ preserva a função hamiltoniana $H$.

Prova. Para isto, devemos verificar que $\mathcal{L}_{X_{H}} H=0$. Temos que

$$
\mathcal{L}_{X_{H}} H=i_{X_{H}} d H=i_{X_{H}} i_{X_{H}} \omega=0 .
$$




\section{Exemplos}

1. O cilindro $\left(\mathbb{S}^{1} \times \mathbb{R}, d \theta \wedge d h\right)$ com coordenadas $(\theta, h)$ e $X(\theta, h)=\frac{\partial}{\partial h}$. $\mathrm{O}$ campo vetorial $X$ é simplético, mas não é hamiltoniano.

i) O campo vetorial $X$ é simplético. De fato,

$$
\begin{aligned}
i_{X} \omega & =i_{\frac{\partial}{\partial h}}(d \theta \wedge d h) \\
& \left.=i_{\frac{\partial}{\partial h}} d \theta\right) d h-d \theta i_{\frac{\partial}{\partial h}}(d h) \\
& =\underbrace{d \theta\left(\frac{\partial}{\partial h}\right)}_{0} d h-\underbrace{d h\left(\frac{\partial}{\partial h}\right)}_{1} d \theta=-d \theta .
\end{aligned}
$$

Portanto, $d\left(i_{X} \omega\right)=-d(d \theta)=0$, logo, $X$ é simplético.

ii) O campo vetorial $X$ não é hamiltoniano. Significa que $i_{X} \omega=-d \theta$ não é exata. Considere o caminho fechado em $\mathbb{S}^{1} \times \mathbb{R}$ dado por $\gamma$ tal que $\gamma:[0,2 \pi] \rightarrow \mathbb{S}^{1}, \operatorname{com} \gamma(t)=(\cos t, \sin t)$ e $\theta: V \rightarrow \mathbb{R}$ a função ângulo, $V$ aberto de $\mathbb{S}^{1}$.

Vamos supor, por absurdo, que $i_{X} \omega=-d \theta$ seja exata. Desta forma, queremos mostrar: $\int_{\gamma} d \theta \neq 0$, encontrando assim uma contradição com a hipótese. De fato,

$$
\int_{0}^{2 \pi} d \theta=2 \pi \neq 0
$$

Temos que, pelo Teorema de Stokes, a integral em torno do caminho fechado é nula, portanto, a igualdade acima contraria o Teorema.

2. O conjunto $\left(\mathbb{R}^{2} \backslash\{0\}, d x \wedge d y\right)$ com coordenadas $(x, y)$ e campo vetorial $X(x, y)=a(x, y) \frac{\partial}{\partial x}+b(x, y) \frac{\partial}{\partial y}$ para a base $\left\{\frac{\partial}{\partial x}, \frac{\partial}{\partial y}\right\}$ fixada.

i) O campo vetorial $X$ é simplético em $\mathbb{R}^{2} \backslash\{0\}$ se, e somente se, $\frac{\partial a}{\partial x}(x, y)=-\frac{\partial b}{\partial y}(x, y)$. Temos

$$
\begin{aligned}
i_{X} \omega_{0} & =i_{X}(d x \wedge d y) \\
& =\left(i_{X} d x\right) \wedge d y-d x \wedge\left(i_{X} d y\right) .
\end{aligned}
$$

Como

$$
\left\{\begin{array}{l}
i_{X} d x=d x(X)=d x\left(a \frac{\partial}{\partial x}+b \frac{\partial}{\partial y}\right)=a \\
i_{X} d y=d y(X)=d y\left(a \frac{\partial}{\partial x}+b \frac{\partial}{\partial y}\right)=b
\end{array}\right.
$$


então $i_{X} \omega_{0}=a d y-b d x$. Além disso, a 1 -forma $i_{X} \omega_{0}$ é fechada se $d\left(i_{X} \omega_{0}\right)=0$. Assim,

$$
\begin{aligned}
d(a(x, y) d y-b(x, y) d x) & =\frac{\partial a}{\partial x} d x \wedge d y-\frac{\partial b}{\partial x} d x \wedge d x+\frac{\partial a}{\partial y} d y \wedge d y-\frac{\partial b}{\partial y} d y \wedge d x \\
& =\frac{\partial a}{\partial x} d x \wedge d y+\frac{\partial b}{\partial y} d x \wedge d y \\
& =\left(\frac{\partial a}{\partial x}+\frac{\partial b}{\partial y}\right) d x \wedge d y
\end{aligned}
$$

porque $d x \wedge d x=d y \wedge d y=0$. Portanto,

$$
d\left(i_{X} \omega_{0}\right)=0 \Leftrightarrow \frac{\partial a}{\partial x}=-\frac{\partial b}{\partial y} .
$$

Logo, $X$ é simplético se, e somente se, $\frac{\partial a}{\partial x}(x, y)=-\frac{\partial b}{\partial y}(x, y)$.

Tomemos, por exemplo, $a(x, y)=\frac{x}{x^{2}+y^{2}}$ e $b(x, y)=\frac{y}{x^{2}+y^{2}}$. Então

$\frac{\partial a}{\partial x}=\frac{x^{2}+y^{2}-2 x \cdot x}{\left(x^{2}+y^{2}\right)^{2}}=\frac{y^{2}-x^{2}}{\left(x^{2}+y^{2}\right)^{2}}$,

$\frac{\partial b}{\partial y}=\frac{x^{2}+y^{2}-2 y \cdot y}{\left(x^{2}+y^{2}\right)^{2}}=\frac{x^{2}-y^{2}}{\left(x^{2}+y^{2}\right)^{2}}$.

Logo $\frac{\partial a}{\partial x}=-\frac{\partial b}{\partial y}$. Desta forma, $i_{X} \omega_{0}$ é fechada para as funções $a(x, y)$ e $b(x, y)$ escolhidas.

ii) O campo vetorial $X: U \rightarrow \mathbb{R}^{2}$ não é hamiltoniano para as mesmas funções $a(x, y)=\frac{x}{x^{2}+y^{2}}$ e $b(x, y)=\frac{y}{x^{2}+y^{2}}$ do item anterior.

Seja $U \subset \mathbb{R}^{2} \backslash\{0\}$ um aberto de $\mathbb{R}^{2} \backslash\{0\}$ que contém uma circunferência $\gamma$ de raio $r>0$, centrada na origem.

Pergunta: Será que existe uma função diferenciável $f: U \rightarrow \mathbb{R}$ tal que $i_{X} \omega=d f ?$

Vamos supor, por absurdo, que a contração da forma $\omega$ pelo campo $X$ seja exata. Como $i_{X} \omega_{0}=a(x, y) d y-b(x, y) d x$ e $\gamma(t)=($ rcost, rsent), $t \in[0,2 \pi]$, temos

$$
\begin{aligned}
a(x, y) d y-b(x, y) d x & =\frac{x}{x^{2}+y^{2}} d y-\frac{y}{x^{2}+y^{2}} d x \\
& =\frac{r \cos t}{r^{2}}(\sin t d r+r \cos t d t)-\frac{r \sin t}{r^{2}}(\cos t d r-r \sin t d t) \\
& =d t
\end{aligned}
$$


e $\int_{0}^{2 \pi} d t=2 \pi \neq 0$. Como no exemplo (ii), pelo Teorema de Stokes temos uma contradição. Logo, $X$ não é hamiltoniano para as funções $a(x, y)$ e $b(x, y)$ escolhidas.

3. Sejam a 2-esfera $\left(\mathbb{S}^{2}, d \theta \wedge d h\right)$ e $X=\frac{\partial}{\partial \theta}$.

i) O campo vetorial $X$ é simplético.

Temos

$$
i_{X} \omega=i_{\frac{\partial}{\partial \theta}}(d \theta \wedge d h)=(d \theta \wedge d h)\left(\frac{\partial}{\partial \theta}\right)=d h,
$$

portanto, $d\left(i_{X} \omega\right)=d(d h)=0$. Assim, $i_{X} \omega$ é fechada, logo $X$ é simplético.

ii) O campo $X$ é hamiltoniano.

Temos que $i_{X} \omega=d h$ e $h: \mathbb{S}^{2} \rightarrow \mathbb{R}$ é suave.

Observação: O movimento gerado por este campo vetorial é a rotação ao redor do eixo vertical, o que naturalmente preserva a área e a altura.

4. Sejam o 2-toro $\left(\mathbb{T}^{2}, d \theta_{1} \wedge d \theta_{2}\right), X_{1}=\frac{\partial}{\partial \theta_{1}}$ e $X_{2}=\frac{\partial}{\partial \theta_{2}}$.

i) Os campos vetoriais $X_{1}$ e $X_{2}$ são simpléticos.

De fato, temos

$i_{X_{1}} \omega=i_{\frac{\partial}{\partial \theta_{1}}}\left(d \theta_{1} \wedge d \theta_{2}\right)=\overbrace{\left(i_{\frac{\partial}{\partial \theta_{1}}} d \theta_{1}\right)}^{1} \wedge d \theta_{2}-d \theta_{1} \wedge \overbrace{i_{\frac{\partial}{\partial \theta_{1}}\left(d \theta_{2}\right)}^{0}}^{0}=d \theta_{2}$.

Portanto, $d\left(i_{X_{1}} \omega\right)=d\left(d \theta_{2}\right)=0 \Rightarrow i_{X_{1}} \omega$ é fechada e assim $X_{1}$ é simplético.

E também

$i_{X_{2}} \omega=i_{\frac{\partial}{\partial \theta_{2}}}\left(d \theta_{1} \wedge d \theta_{2}\right)=\overbrace{\left(i_{\frac{\partial}{\partial \theta_{2}}} d \theta_{1}\right)}^{0} \wedge d \theta_{2}-d \theta_{1} \wedge \overbrace{i_{\frac{\partial}{\partial \theta_{2}}}\left(d \theta_{2}\right)}^{1}=-d \theta_{1}$. Portanto $d\left(i_{X_{2}} \omega\right)=-d\left(d \theta_{1}\right)=0 \Rightarrow i_{X_{2}} \omega$ é fechada e assim $X_{2}$ é simplético.

ii) Os campos vetoriais $X_{1}$ e $X_{2}$ não são hamiltonianos.

Como as contrações são fechadas, $X_{1}$ e $X_{2}$ são simpléticos e, pelo lema 2.15, são localmente hamiltonianos. Porém, todo o campo de vetores localmente hamiltoniano que não se anula sobre uma variedade 
simplética compacta não pode ser hamiltoniano. De fato, se $X_{1}=X_{H}$ para alguma função $H$ em $\mathbb{T}^{2}$, temos que $X_{1}$ tem um zero porque $\mathrm{H}$ tem pontos críticos sobre a variedade compacta $\mathbb{T}^{2}$. Assim, $X_{1}$ não pode ser hamiltoniano. Analogamente, temos o mesmo resultado para o campo de vetores $X_{2}$. 


\section{Sistemas Integráveis}

Neste capítulo recordamos um importante conceito da teoria dos grupos de Lie que será usado até o final deste trabalho, a álgebra de Lie. Também trataremos do sistema hamiltoniano, cuja teoria leva ao enunciado do importante Teorema de Arnold-Liouville, que caracteriza o espaço de fases de um sistema integrável.

Lembremos que uma álgebra de Lie $^{a}$ é um espaço vetorial $\mathfrak{g}$ com uma operação [., .] : $\mathfrak{g} \times \mathfrak{g} \rightarrow \mathfrak{g}$ que satisfaz:

(i) a anti-simetria:

$$
[x, y]=-[y, x], \forall x, y \in \mathfrak{g}
$$

(ii) a bilinearidade:

$$
[a x+b y, z]=a[x, z]+b[y, z], \forall x, y, z \in \mathfrak{g} ; a, b \in \mathbb{R}
$$

(iii) a Identidade de Jacobi:

$$
[x,[y, z]]+[y,[z, x]]+[z,[x, y]]=0, \forall x, y, z \in \mathfrak{g}
$$

Seja $(M, \omega)$ variedade simplética. O espaço $\mathfrak{X}(M)$ com o colchete de Lie [ , ] definido em (2.1) é um exemplo de álgebra de Lie.

Agora, considere o campo vetorial hamiltoniano $X_{H}$ na variedade simplética $(M, \omega)$ com a função hamiltoniana $H \in C^{\infty}(M)$.

Definição 3.1 Seja $(M, \omega)$ uma variedade simplética. O colchete de Poisson de duas funções $f, g \in C^{\infty}(M)$ é a operação $\{.,\}:. C^{\infty}(M) \times C^{\infty}(M) \rightarrow C^{\infty}(M)$ que associa cada par $(f, g)$ à $\{f, g\}$ definida da seguinte forma:

$$
\{f, g\}:=\omega\left(X_{f}, X_{g}\right)=X_{g}(f)
$$

em coordenadas de Darboux $\left(x_{1}, \ldots, x_{n}, y_{1}, \ldots, y_{n}\right)$, escrevemos

$$
\{f, g\}=\sum_{i=1}^{n}\left(\frac{\partial f}{\partial x_{i}} \frac{\partial g}{\partial y_{i}}-\frac{\partial g}{\partial x_{i}} \frac{\partial f}{\partial y_{i}}\right) \text {. }
$$

${ }^{a}$ Para aprofundar, consulte [10]. 
Definição 3.2 Uma álgebra de Poisson é uma álgebra de Lie com um produto associativo para o qual a Regra de Leibniz é válida, isto é, satisfaz a propriedade:

$$
\{f, g h\}=\{f, g\} \cdot h+g \cdot\{f, h\}, \forall f, g, h \in C^{\infty}(M) .
$$

Definição 3.3 $M$ é dita variedade de Poisson se $\left(C^{\infty}(M),\{.,\}.\right)$ é uma álgebra de Poisson. Neste caso, dizemos que $\{.,$.$\} é uma estrutura de Poisson em M.$

Proposição 3.4 Toda variedade simplética é uma variedade de Poisson.

Prova. Sejam $(M, \omega)$ variedade simplética e $f, g, h \in C^{\infty}(M)$. Esta proposição signica que o colchete de Poisson é um colchete de Lie que satisfaz a Regra de Leibniz. De fato temos, pela definição 3.1 e pela proposição 2.9, que

$$
\{f, g\}:=\omega\left(X_{f}, X_{g}\right)=X_{g}(f)=\mathcal{L}_{X_{g}}(f) .
$$

Verifiquemos as três condições para que seja um colchete de Lie, seguida da Regra de Leibniz:

i) anti-simetria:

$$
\{f, g\}=\omega\left(X_{f}, X_{g}\right)=-\omega\left(X_{g}, X_{f}\right)=-\{g, f\}
$$

ii) bilinearidade:

$$
\begin{aligned}
\{a f+b g, h\} & =X_{h}(a f+b g) \\
& =X_{h}(a f)+X_{h}(b g) \\
& =a X_{h}(f)+b X_{h}(g) \\
& =a\{f, h\}+b\{g, h\}, \forall a, b \in \mathbb{R},
\end{aligned}
$$

iii) identidade de Jacobi:

$$
\begin{aligned}
\{f,\{g, h\}\}+\{g,\{h, f\}\} & =-X_{f}(\{g, h\})-X_{g}(\{h, f\} \\
& =-X_{f}\left(X_{h}(g)\right)-X_{g}\left(X_{f}(h)\right) \\
& =X_{f}\left(X_{g}(h)\right)-X_{g}\left(X_{f}(h)\right) \\
& =X_{h}\left(X_{g}(f)\right) \\
& =X_{h}(\{f, g\}) \\
& =-\{h,\{f, g\}\}
\end{aligned}
$$


iv) Regra de Leibniz:

$$
\begin{aligned}
\{f, g h\} & =\omega\left(X_{f}, X_{g h}\right) \\
& =X_{g h}(f)=-X_{f}(g h) \\
& =-\left(X_{f}(g) \cdot h+g \cdot X_{f}(h)\right) \\
& =-\left(\omega\left(X_{g}, X_{f}\right) \cdot h+g \cdot \omega\left(X_{h}, X_{f}\right)\right) \\
& =\omega\left(X_{f}, X_{g}\right) \cdot h+g \cdot \omega\left(X_{f}, X_{h}\right) \\
& =\{f, g\} h+g\{f, h\} .
\end{aligned}
$$

Lema 3.5 Sejam $X_{f}, X_{g}$ campos hamiltonianos associados às funções hamiltonianas $f$ e $g$, respectivamente ${ }^{b}$. Então $X_{\{f, g\}}=-\left[X_{f}, X_{g}\right]$, sendo [., .] o colchete de Lie.

Prova. Para quaisquer $f, g, h \in C^{\infty}(M)$, temos

$$
\begin{aligned}
{\left[X_{f}, X_{g}\right] h } & =X_{f}\left(X_{g} h\right)-X_{g}\left(X_{f} h\right) \\
& =X_{f}(\{h, g\})-X_{g}(\{h, f\}) \\
& =\{\{h, g\}, f\}-\{\{h, f\}, g\} \\
& =\{g,\{h, f\}\}+\{f,\{g, h\}\} \\
& =-\{h,\{f, g\}\} \\
& =-X_{\{f, g\}} h .
\end{aligned}
$$

Proposição 3.6 As álgebras de Lie $C^{\infty}(M)$ e $\mathfrak{X}(M)$ são anti-homomorfas.

Prova. Seja $L:\left(C^{\infty}(M),\{.,\}.\right) \rightarrow(\mathfrak{X}(M),[.,]$.$) , que associa cada função hamil-$ toniana $H$ a um campo vetorial hamiltoniano $X_{H}$.

- $L$ é linear: Devemos mostrar que para $F, H \in C^{\infty}(M)$ e $a \in \mathbb{R}$, temos $L(F+a H)=L(F)+a L(H)$.

De fato, seja $K \in C^{\infty}(M)$

${ }^{b}$ Isto é, $X_{f}$ e $X_{g}$ são campos tais que $i_{X_{f}} \omega=d f$ e $i_{X_{g}} \omega=d g$. 


$$
\begin{aligned}
L(F+a H)(K) & =X_{F+a H}(K) \\
& =\{K, F+a H\} \\
& =\{K, F\}+a\{K, H\} \\
& =X_{F}(K)+a X_{H}(K) \\
& =L(F)(K)+a L(H)(K) .
\end{aligned}
$$

. $L$ é anti-homomorfismo: Ou seja, $L(\{F, H\})=-[L(F), L(H)]$, para $F, H \in C^{\infty}(M)$.

Equivalentemente, seja $K \in C^{\infty}(M)$, vale

$$
\begin{aligned}
{[L(F), L(H)](K) } & =\left[X_{F}, X_{H}\right](K) \\
& =X_{F} X_{H}(K)-X_{H} X_{F}(K) \\
& =X_{F}(\{K, H\})-X_{H}(\{K, F\}) \\
& =\{\{K, H\}, F\}-\{\{K, F\}, H\} \\
& =\{\{K, H\}, F\}+\{\{F, K\}, H\} \\
& =-\{K,\{F, H\}\} \\
& =-X_{\{F, H\}}(K) \\
& =-L(\{F, H\})(K) .
\end{aligned}
$$

Definição 3.7 Um sistema hamiltoniano é uma tripla $(M, \omega, H)$, onde $(M, \omega)$ é uma variedade simplética e $H \in C^{\infty}(M)$ é uma função hamiltoniana.

Seja $X$ campo vetorial na variedade suave $M$. Se $\gamma: J \longrightarrow M$ é uma curva suave para cada $t \in J$, chamamos curva integral ou caminho ${ }^{c}$ de $X$ a curva diferenciável $\gamma$ tal que $\gamma^{\prime}(t)=X_{\gamma(t)}, \forall t \in J$.

Definição 3.8 Uma integral primeira ou constante de movimento de um campo $X$ é uma função $H$ tal que $X(H)=0$, ou seja, $H$ é constante em relação ao campo $X$ ou curva integral de $X$.

Teorema 3.9 São equivalentes as afirmações:

(i) $\{f, H\}=0$.

${ }^{c}$ Também chamamos de curva integral ou caminho de uma equação diferencial $F\left(x, y, y^{\prime}\right)=0$ ao gráfico de solução $y=\phi(h)$ dessa equação diferencial. 
(ii) $f$ é uma integral primeira de um campo hamiltoniano $X_{H}$.

(iii) $H$ é constante ao longo das curvas integrais do campo $X_{f}$.

Prova. $(i) \Rightarrow(i i)$ :

Sejam $H \in C^{\infty}(M)$ e $\phi_{H}$ o fluxo do campo hamiltoniano associado a $X_{H}$. Então

$$
\frac{d}{d t}\left(f \circ \phi_{H}\right)(t, p)=\{f, H\}\left(\phi_{H}(t, p)\right), \forall(t, p) .
$$

Se $\{f, H\}=0$, então $\frac{d}{d t}\left(f \circ \phi_{H}\right)(t, p)=0$. Logo, $f$ é constante ao longo das curvas integrais de $X_{H}$.

$(i i) \Rightarrow(i)$ :

Assumimos $f$ constante ao longo das curvas integrais de $X_{H}$.

$\Rightarrow\{f, H\}=f\{1, H\}$.

Pela Regra de Leibniz,

$\{1, H\}=\{1.1, H\}=1\{1, H\}+1\{1, H\}=2\{1, H\}$

$\Rightarrow\{1, H\}=0$.

Logo, $\{f, H\}=0$.

$($ ii $) \Leftrightarrow($ iii $)$ :

Pela antissimetria de $\{f, H\}$.

Nota: Como consequência do teorema anterior, toda função $H$ é uma integral primeira de seu próprio campo hamiltoniano, já que, pela antissimetria, temos $X_{H} H=\{H, H\}=0$.

Teorema 3.10 (Poisson) Sejam $f, g, h \in C^{\infty}(M), M$ uma variedade de Poisson. Se $\{g, f\}=0$ e $\{h, f\}=0$, então $\{\{g, h\}, f\}=0$.

Prova. Segue da identidade de Jacobi.

Definição 3.11 Sejam $(M, \omega)$ variedade simplética, $H \in C^{\infty}(M)$ função hamiltoniana e $f_{1}=H, f_{2}, \ldots, f_{n}$ constantes de movimento. $O$ conjunto $\left\{f_{1}, \ldots, f_{n}\right\}$ é dito em involução se $\left\{f_{i}, f_{j}\right\}=0$ para todo $1 \leq i, j \leq n$.

Definição 3.12 As funções $\left\{f_{1}, \ldots, f_{n}\right\}$ em $M$ são ditas independentes se suas diferenciais $\left(d f_{1}\right)_{p}, \ldots,\left(d f_{n}\right)_{p}$ são linearmente independentes para todo $p$ em algum subconjunto denso aberto de $M$. 
Definição 3.13 Um sistema hamiltoniano $\left(M^{2 n}, \omega, H\right)$ é dito completamente integrável se possui $n$ constantes de movimento independentes e em involução, ou seja, se possuir tantas integrais primeiras quanto possível.

Definição 3.14 Seja $(V, \omega)$ um espaço vetorial. O anulador de um subconjunto $Y \subset V$ é o subespaço

$$
Y^{0}:=\left\{f \in V^{*} \mid f(y)=0, \forall y \in Y\right\} .
$$

Definição 3.15 Seja $(V, \omega)$ um espaço vetorial simplético. O ortogonal (ou complemento) simplético de um subespaço linear $Y \subset V$ é definido como o subespaço

$$
Y^{\omega}:=\{v \in V \mid \omega(v, u)=0, \forall u \in Y\}
$$

Proposição $3.16 \mathrm{Em}$ um espaço vetorial simplético $(V, \omega)$, dado o subespaço $Y \in V$, temos

$$
\operatorname{dim} Y+\operatorname{dim} Y^{\omega}=\operatorname{dim} V
$$

Prova. Seja $\phi: V \longrightarrow V^{*}$ a função linear definida por $\phi(x)(y)=\omega(x, y)$. Como $\omega$ é uma forma simplética, é portanto não degenerada e $\phi$ é um isomorfismo. Além disso temos que $\phi\left(Y^{\omega}\right)=Y^{0}$ é o anulador de $Y$. Assim

$$
\operatorname{dim} Y^{\omega}=\operatorname{dim} Y^{0}=\operatorname{dim} V-\operatorname{dim} Y .
$$

Lema 3.17 Seja $Y \subset V,(V, \omega)$ espaço vetorial simplético, então $\left(Y^{\omega}\right)^{\omega}=Y$.

Prova. Segue, da definição que $Y \subset\left(Y^{\omega}\right)^{\omega}$. Da proposição anterior, temos

$$
\operatorname{dim} V=\operatorname{dim} Y+\operatorname{dim} Y^{\omega}=\operatorname{dim} Y^{\omega}+\operatorname{dim}\left(Y^{\omega}\right)^{\omega} .
$$

Logo, $\operatorname{dim} Y=\operatorname{dim}\left(Y^{\omega}\right)^{\omega}$ e, portanto, $Y=\left(Y^{\omega}\right)^{\omega}$.

Definição 3.18 Um subespaço $Y \subset V$ de um espaço vetorial simplético $(V, \omega)$ é chamado lagrangiano ou de isotrópico ${ }^{d}$ se $Y=Y^{\omega}$ ou $Y \subset Y^{\omega}$, respectivamente.

${ }^{d}$ Veja exemplos em [11]. 
Definição 3.19 Seja $(M, \omega)$ uma variedade simplética. $N \subset M$ é dita subvariedade lagrangiana (ou subvariedade isotrópica) se, para todo $y \in N$, o subespaço $T_{y} N \subset\left(T_{y} M, \omega_{y}\right)$ é lagrangiano (ou isotrópico).

Nota: A subvariedade $N$ da variedade simplética $\left(M^{2 n}, \omega\right)$ é lagrangiana se $N$ é $n$-dimensional e se $i^{*} \omega=0$, onde $i: N \hookrightarrow M$ é a inclusão e $\operatorname{dim} M=2 \operatorname{dim} N$.

Teorema 3.20 (Arnold-Liouville,[12]) Seja $\left(M^{2 n}, \omega, H\right)$ um sistema integrável com $n$ integrais primeiras $f_{1}=H, f_{2}, \ldots, f_{n}$. Seja $f=\left(f_{1}, \ldots, f_{n}\right)$ própria com fibras conexas e considere $c \in \mathbb{R}^{n}$ um valor regular de $f$. Então $f^{-1}(c)$ é uma subvariedade lagrangiana de $M^{2 n}$. Temos:

(i) o conjunto de nível $f^{-1}(c)$ é invariante para toda função $f_{1}, \ldots, f_{n}$, isto é, toda a curva integral de $X_{f_{i}}$ que começa em $p \in f^{-1}(c)$ permanece em $f^{-1}(c)$. Além disso, $f^{-1}(c)$ é difeomorfo a $\mathbb{T}^{n}$ com coordendas $\theta_{1}, \cdots, \theta_{n}$ (chamadas de coordenadas ângulo),

(ii) existem $\psi_{1}, \cdots, \psi_{n}$ (chamadas de coordenadas ação), de forma que $\left(\theta_{1}, \cdots, \theta_{n}, \psi_{1}, \cdots, \psi_{n}\right)$ é uma carta de Darboux em $M$ e

$$
\omega=\sum_{i=1}^{n} d \theta_{i} \wedge d \psi_{i}
$$

Nota: Temos que a subvariedade é langrangiana por possui dimensão $n$ e seu fibrado tangente é isotrópico (o fibrado está contido em seu complemento simplético). 


\section{4}

\section{Ações de Grupos Sobre Variedades}

Muitos problemas matemáticos apresentam mais informações que o necessário, isto é, simetrias. No estudo da geometria, as simetrias são codificadas por ações de grupos. O objetivo será poder tornar algumas informações numa linguagem mais simples através das ações, tendo como ferramenta a forma simplética.

\section{1}

\section{Ações de grupos}

Definição 4.1 Seja $(G, \cdot)$ um grupo e $S$ um conjunto. Dizemos que $G$ atua sobre $S$ ou $G \curvearrowright S$ (à esquerda) se existe uma aplicação $\alpha: G \times S \longrightarrow S$, com $\alpha(g, s)=g \cdot s$ tal que

(i) $\alpha(e, s)=e \cdot s=s, \forall s \in S$,

(ii) $\alpha(g, \alpha(h, s))=\alpha(g h, s)$, ou seja, $g \cdot(h \cdot s)=(g h) \cdot s, \forall g, h \in G, s \in S$.

Chamamos a aplicação $\alpha$ de ação do grupo $G$ sobre o conjunto $S$.

Definição 4.2 Seja $G$ um grupo de Lie. A ação de $G$ em uma variedade $M$ é um homomorfismo de grupo dado por

$$
\psi: G \longrightarrow \operatorname{Diff}(M) \text { tal que } \psi(g)=\psi_{g}
$$

que associa cada elemento do grupo de Lie a um elemento do grupo de difeomorfismos da variedade $M$. Dizemos que a ação é suave se a aplicação $e v_{\psi}: M \times G \longrightarrow G$ definida por $e v_{\psi}(p, g)=\psi_{g}(p)$ é suave. Nota: Sempre assumiremos que a ação é suave.

Definição 4.3 Sejam $(M, \omega)$ variedade simplética, G grupo de Lie e considere a ação

$$
\begin{array}{cl}
G \times M & \longrightarrow \\
(g, p) & \longmapsto \\
&
\end{array}
$$


o estabilizador ou grupo de isotropia de p é definido por

$$
G_{p}:=\{g \in G \mid g \cdot p=p\} \subseteq G
$$

A órbita de $G$ em $p$ é definida por

$$
G \cdot p:=\{g \cdot p \mid g \in G\} \subseteq M
$$

Denotamos o conjunto de todas as órbitas por $M / G:=\{G \cdot p \mid p \in M\}$.

Definição 4.4 Uma ação $\psi: G \times M \longrightarrow M$ é dita

i) transitiva: se houver apenas uma órbita, isto é, se existir $p \in M$ tal que $G \cdot p=M$

ii) livre: se $G_{p}=\{e\}, \forall p \in M$ e localmente livre se todos os estabilizadores são discretos.

Um campo vetorial completo é um campo cujas curvas integrais existem para todo o tempo.

Definição 4.5 Sejam $G$ um grupo de Lie e $M$ uma variedade diferenciável. Suponha $G \curvearrowright M$ pela ação $\psi: G \times M \longrightarrow M$. Para todo $\xi \in \mathfrak{g}$, o campo fundamental associado em $M$ é definido por

$$
X^{\xi}(p):=\left.\frac{d}{d t} \psi(\exp (t \xi), p)\right|_{t=0}
$$

com $p \in M$, onde a função $\mathfrak{g} \ni \xi \longmapsto X^{\xi}$ é chamada ação infinitesimal associada a $\psi$.

4.2

Ações Hamiltonianas

Definição 4.6 Dado G um grupo de Lie e $\mathfrak{g}$ sua ágebra de Lie, considere a ação $\psi_{g}: G \longrightarrow G$ com $\psi_{g}(a)=g a g^{-1}$. Chamamos de ação adjunta ou representação adjunta $\left(A d_{g}\right)$ à derivada da aplicação $\psi_{g}$ na identidade, isto é

$$
\begin{aligned}
A d_{g}:=d_{e} \psi_{g}: G \times \mathfrak{g} & \longrightarrow \mathfrak{g} \\
(g, \xi) & \longmapsto A d_{g}(\xi)=\frac{d}{d t}\left(g \exp (t \xi) g^{-1}\right) .
\end{aligned}
$$


Como definimos uma ação do grupo de Lie em sua álgebra de Lie, também podemos definir uma ação do grupo no dual da sua álgebra de Lie:

Definição 4.7 Chamamos de ação coadjunta do grupo de Lie $G$ à aplicação $A d^{*}: G \times \mathfrak{g}^{*} \longrightarrow \mathfrak{g}^{*}$ definida por

$$
A d^{*}(g, \eta)=A d_{g}^{*}(\eta):=\eta \circ A d\left(g^{-1}\right) .
$$

Definição 4.8 Sejam $(M, \omega)$ e $G$ variedade simplética e grupo de Lie, respectivamente e considere a ação $\psi: G \longrightarrow \operatorname{Diff}(M)$. A aplicação $\psi$ é uma ação simplética se atua por simplectomorfismos, ou seja, se

$$
\psi: G \longrightarrow \operatorname{Sympl}(M, \omega) \subset \operatorname{Diff}(M)
$$

sendo Sympl $(M, \omega)$ o grupo de simplectomorfismos da variedade $(M, \omega)$.

\section{Exemplos}

(i) Na 2-esfera simplética $\left(\mathbb{S}^{2}, d \theta \wedge d h\right)$, em coordenadas cilíndricas, o grupo a 1parâmetro de difeomorfismos ${ }^{a}$ dado pela rotação em torno do eixo vertical, $\psi_{t}(\theta, h)=(\theta+t, h), t \in \mathbb{R}$ é a ação simplética do grupo $\mathbb{S}^{1} \simeq \frac{\mathbb{R}}{\langle 2 \pi\rangle}$, uma vez que preserva a forma de área $(d \theta \wedge d h)$. De fato, considerando a ação

$$
\begin{aligned}
\psi_{\theta+t}: \mathbb{S}^{1} \times \mathbb{S}^{2} & \longrightarrow \mathbb{S}^{2} \\
(t,(\theta, h)) & \longmapsto t \cdot(\theta, h)=(\theta+t, h)
\end{aligned}
$$

e a forma simplética $\omega=d \theta \wedge d h$, temos

$$
\begin{aligned}
\psi_{\theta+t}^{*} \omega & =d(\theta+t) \wedge d(h) \\
& =(d \theta+d t) \wedge d h \\
& =d \theta \wedge d h=\omega .
\end{aligned}
$$

(ii) No 2-toro simplético $\left(\mathbb{T}^{2}, d \theta_{1} \wedge d \theta_{2}\right)$, os grupos a 1-parâmetro de difeomorfismos dados pela rotação em torno de cada círculo $\psi_{1, t}\left(\theta_{1}, \theta_{2}\right)=\left(\theta_{1}+t, \theta_{2}\right)$

${ }^{a}$ Temos que a família $\left\{\rho_{t} \mid t \in \mathbb{R}\right\}$ é chamada grupo 1-parâmetro de difeomorfismos da variedade $M$ e denotado por

$$
\rho_{t}=\exp (t X), X \in T_{e} M
$$


e $\psi_{2, t}\left(\theta_{1}, \theta_{2}\right)=\left(\theta_{1}, \theta_{2}+t\right), t \in \mathbb{R}$, são ações simpléticas de $\mathbb{S}^{1}$. De fato, temos para $\omega=d \theta_{1} \wedge d \theta_{2}$

$$
\begin{aligned}
\psi_{1, t}^{*} \omega & =d\left(\theta_{1}+t\right) \wedge d \theta_{2} \\
& =\left(d \theta_{1}+d t\right) \wedge d \theta_{2} \\
& =d \theta_{1} \wedge d \theta_{2}=\omega .
\end{aligned}
$$

Analogamente, $\psi_{2, t}^{*} \omega=\omega$.

Definição 4.9 Sejam $M, N$ variedades e $G$ um grupo de Lie. Considere as ações $\phi$ e $\psi$ de $G$ sobre $M$ e $N$, respectivamente, e $f: M \longrightarrow N$ uma aplicação diferenciável. Dizemos que $f$ é equivariante com respeito a essas ações se, para todo $g \in G$, temos

$$
f \circ \phi_{g}=\psi_{g} \circ f
$$

isto é, se $f$ preserva a ação de grupo.

Definição 4.10 Sejam $(M, \omega)$ uma variedade simplética, $G$ um grupo de Lie com a ação $\psi: G \longrightarrow \operatorname{Diff}(M)$ e $\mathfrak{g}=\operatorname{Lie}(G)$, sendo $\mathfrak{g}^{*}$ o seu espaço vetorial dual. $A$ ação $\psi$ é chamada de ação hamiltoniana se houver uma aplicação $\mu: M \longrightarrow \mathfrak{g}^{*}$ satisfazendo:

(i) para cada $X \in \mathfrak{g}$, seja $\mu^{X}: M \longrightarrow \mathbb{R}$ tal que $\mu^{X}(p):=\langle\mu(p), X\rangle$ a componente de $\mu$ ao longo de $X$ e seja $X^{\sharp}$ o campo vetorial em $M$ gerado pelo subgrupo a 1-parâmetro $\{\exp (t X) \mid t \in \mathbb{R}\} \subseteq G$. Então

$$
d \mu^{X}=i_{X}^{\sharp \omega}
$$

ou seja, a função $\mu^{X}$ é a função hamiltoniana para o campo vetorial $X^{\sharp}$,

(ii) a aplicação $\mu$ é equivariante em relação à ação $\psi$ de $G$ em $M$ dada e a ação coadjunta $A d^{*}$ de $G$ em $\mathfrak{g}^{*}$, ou seja,

$$
\mu \circ \psi_{g}=A d_{g}^{*} \circ \mu, \forall g \in G
$$

Chamamos a $(M, \omega, G, \mu)$ de $G$-espaço hamiltoniano e $\mu$ de aplicação momento.

Nota: Além disso, sendo $\mu$ a aplicação momento, qualquer uma de suas translações $\mu+C, C \in \mathfrak{g}^{*}$, é também uma aplicação momento para essa ação, fato esse vindo pela integração (onde é inserida a constante $C$ ). 
Proposição 4.11 Seja $(M, \omega)$ variedade simplética. Os campos vetoriais simpléticos completos em $M$ estão em correspondência biunívoca com ações simpléticas de $\mathbb{R}$ em $M$. Similarmente, existe uma bijeção entre os campos vetoriais hamiltonianos completos em $M$ com as ações hamiltonianas de $\mathbb{R}$ em $M$.

Prova.

(i) $\{$ Campos vetoriais simpléticos completos $\} \leftrightarrow\{$ Ações simpléticas de $\mathbb{R}$ em $M\}$.

Seja $X$ um campo vetorial simplético completo em $M$. Para cada $t \in \mathbb{R}$ temos uma correspondência biunívoca com a aplicação exponencial calculada por $\exp (t X) \in \mathbb{R}$. Assim, existe $\psi_{\exp (t X)}$ ação de $\mathbb{R}$ em $M$ tal que

$$
\begin{aligned}
& \psi_{\exp (t X)}: M \longrightarrow M \quad \text { equivale a } \psi: \mathbb{R} \times M \longrightarrow M
\end{aligned}
$$

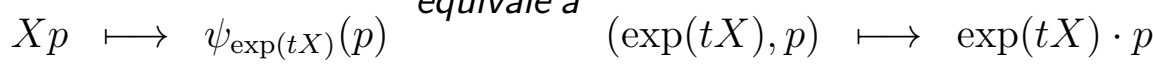

além disso, dada a ação simplética $\psi: \mathbb{R} \longrightarrow \operatorname{Sympl}(M, \omega)$, cada campo vetorial completo é determinado por $X_{p}=\frac{d}{d t} \psi_{t}(p)$.

(ii) \{Campos vetoriais hamiltonianos completos $\} \leftrightarrow\{$ Ações hamiltonianas\}.

A escolha de $X \in T_{e} M$ define biunivocamente cada $\mu^{X}: M \longrightarrow \mathbb{R}$, $\mu^{X}(p)=\langle\mu(p), X\rangle$ e, consequentemente, a aplicação momento $\mu$, que é equivariante por ser uma ação de $\mathbb{R}$ em uma variedade.

Como a ação $\psi$ é hamiltoniana, então existe a função $H: M \longrightarrow \mathbb{R}$ tal que $d H=i_{X} \omega$, onde $X$ é campo vetorial hamiltoniano em $M$ gerado por $\psi$. Logo, $X$ é completo, pois o domínio de $X_{t}$ é $M$, para todo $t \in \mathbb{R}$.

\section{3}

\section{Ações Tóricas Hamiltonianas}

Uma ação tórica hamiltoniana sobre uma variedade simplética $(M, \omega)$ é uma ação hamiltoniana de um toro $\mathbb{T}^{n}$ para $\operatorname{dim} M=2 n$. Ações do toro podem ser vistas como uma coleção de ações do círculo, que comutam entre elas. Nesta seção veremos o caso em que um toro $n$-dimensional atua de forma hamiltoniana sobre uma variedade simplética $2 n$-dimensional e quando podemos classificar esta como uma variedade tórica simplética. 
Definição 4.12 Sejam $\left(M^{2 n}, \omega\right)$ uma variedade simplética e $G=\mathbb{T}^{n}$ o $n$-toro com a ação $\psi: G \longrightarrow \operatorname{Diff}(M)$. Dizemos que $\psi$ é ação tórica hamiltoniana se houver a aplicação $\mu: M \longrightarrow \mathfrak{g}^{*}=\mathbb{R}^{n}$ satisfazendo:

(i) para cada base de vetores $X_{i}$ de $\mathbb{R}^{n}$, a função $\mu^{X_{i}}$ é a função hamiltoniana para $X_{i}^{\sharp}$. Isto é,

$$
d \mu^{X_{i}}=i_{X_{i}^{\sharp} \omega}
$$

(ii) a aplicação $\mu$ é invariante pela ação do toro.

Nota: $\mu$ é invariante pela ação de $\mathbb{T}^{n} \Leftrightarrow \mu(x)=\mu\left(\psi_{g}(x)\right), \forall x \in M, g \in \mathbb{T}^{n}$. Consequência da invariância: $\mathcal{L}_{X^{\sharp}} \mu=i_{X^{\sharp}} d \mu=0$.

\section{Exemplos}

I) Seja $\left(\mathbb{C}, \omega_{0}=\frac{i}{2} d z \wedge d \bar{z}\right)$ e considere a ação do círculo $\mathbb{S}^{1}=\{\xi \in \mathbb{C}|| \xi \mid=1\}$ por rotações:

$$
\begin{aligned}
\psi_{\xi}: \mathbb{S}^{1} \times \mathbb{C} & \longrightarrow \mathbb{C} \\
(\xi, z) & \longmapsto \xi^{k} z
\end{aligned}
$$

para $k \in \mathbb{Z}$ fixo. Vamos calcular a aplicação momento pelos passos seguintes:

\section{. Passo 1: Cálculo do campo fundamental e do campo vetorial $X^{\sharp}$}

Considerando a ação dada como $\mathbb{S}^{1} \curvearrowright \mathbb{R}^{2} \simeq \mathbb{C}$ e a forma $\omega=d x \wedge d y$, temos $\operatorname{Lie}\left(\mathbb{S}^{1}\right) \simeq \mathbb{R} \operatorname{e} \exp (X)=e^{2 \pi i X} \in \mathbb{S}^{1}, \forall X \in \operatorname{Lie}\left(\mathbb{S}^{1}\right)$. Portanto,

$$
\left.\frac{d}{d t} \psi(\exp (t X), z)\right|_{t=0}=\left.\frac{d}{d t} \psi\left(e^{2 \pi i t X}, z\right)\right|_{t=0}
$$

como a ação é $2 \pi$-periódica, então $\psi\left(e^{2 \pi i t X}, z\right)=\psi\left(e^{i t X}, z\right)$. Logo, identificando $\mathbb{R}^{2} \simeq \mathbb{C}$ como habitual $z=x+i y$, temos ${ }^{b}$

$$
\left.\frac{d}{d t} \psi\left(e^{i t X}, z\right)\right|_{t=0}=\left.\frac{d}{d t} e^{i t X k} \cdot z\right|_{t=0}=\left[z e^{i t X k} \cdot i X k\right]_{t=0}=i X k(x+i y)=k(i x-y) .
$$

Assim, temos o campo vetorial $X^{\sharp}$ gerado pelo subgrupo a 1-parâmetro como

$$
X^{\sharp}=-k y \frac{\partial}{\partial x}+k x \frac{\partial}{\partial y} .
$$

\section{. Passo 2: Cálculo da função hamiltoniana $\mu^{X}$ para o campo vetorial $X^{\sharp}$}

${ }^{b}$ Observe que $X \in \operatorname{Lie}\left(\mathbb{S}^{1}\right)$ e que $X$ é base de $\mathbb{R}$, portanto, podemos escolher $X=1$. 
Como $d \mu^{X}=i_{X^{\sharp} \omega}$, então

$$
\begin{aligned}
i_{X^{\sharp} \omega} & =\left(i_{k x \frac{\partial}{\partial y}-k y \frac{\partial}{\partial x}} d x\right) \wedge d y-d x \wedge\left(i_{k x \frac{\partial}{\partial y}-k y \frac{\partial}{\partial x}} d y\right) \\
& =d x\left(k x \frac{\partial}{\partial y}\right) \wedge d y-d x\left(k y \frac{\partial}{\partial x}\right) \wedge d y-d x \wedge d y\left(k x \frac{\partial}{\partial y}\right)-d x \wedge d y\left(-k y \frac{\partial}{\partial x}\right) \\
& =-k y d y-k x d x
\end{aligned}
$$

desta forma, temos $d \mu^{X}=-k y d y-k x d x$. Então

$$
\mu^{X}=-\frac{k}{2}\left(x^{2}+y^{2}\right)+\text { constante }=-\frac{k}{2}|z|^{2}+C .
$$

\section{- Passo 3: Cálculo da aplicação momento}

Como $\mu^{X}(z)=\langle\mu(z), X\rangle$ e $X=1$, então $\mu^{X}(z)=\mu(z)$.

Portanto, a aplicação momento é

$$
\mu(z)=-\frac{k}{2}|z|^{2}+C
$$

II) Seja $\mathbb{T}^{n}=\left\{\left(\xi_{1}, \cdots, \xi_{n}\right) \in \mathbb{C}^{n}|| \xi_{j} \mid=1, \forall j\right\}$ o $n$-toro que atua em $\mathbb{C}^{n}$ pela ação

$$
\begin{aligned}
\psi: \mathbb{T}^{n} \times \mathbb{C}^{n} & \longrightarrow \mathbb{C}^{n} \\
(\xi, z) & \longmapsto\left(\xi_{1}^{k_{1}} z_{1}, \cdots, \xi_{n}^{k_{n}} z_{n}\right) .
\end{aligned}
$$

A ação $\mathbb{T}^{n} \curvearrowright \mathbb{C}^{n}$ equivale à ação $\overbrace{\mathbb{S}^{1} \times \cdots \times \mathbb{S}^{1}}^{n} \curvearrowright \mathbb{R}^{2 n}$. Considerando a 2-forma simplética como $\omega=\sum_{j=1}^{n} d x_{j} \wedge d y_{j}, z_{j}=x_{j}+i y_{j}$ e $k_{j} \in \mathbb{Z}$ fixos, sigamos, novamente, os passos anteriores para determinar a sua aplicação momento:

\section{. Passo 1: Cálculo do campo fundamental e do campo vetorial $X^{\sharp}$}

Temos $\exp (X)=\left(e^{2 \pi i X_{1}}, \cdots, e^{2 \pi i X_{n}}\right)$, para $X=\left(X_{1}, \cdots, X_{n}\right) \in \mathfrak{t}=\mathbb{R}^{n}$. 
Logo, ${ }^{c}$

$$
\begin{aligned}
\left.\frac{d}{d t} \psi(\exp (t X), z)\right|_{t=0} & =\left.\frac{d}{d t} \psi\left(\left(e^{i t X_{1}}, \cdots, e^{i t X_{n}}\right),\left(z_{1}, \cdots, z_{n}\right)\right)\right|_{t=0} \\
& =\left.\frac{d}{d t}\left(e^{i t X_{1} k_{1}} z_{1}, \cdots, e^{i t X_{n} k_{n}} z_{n}\right)\right|_{t=0} \\
& =i\left(X_{1} k_{1} z_{1}, \cdots, X_{n} k_{n} z_{n}\right) \\
& =\left(X_{1} k_{1}\left(i x_{1}-y_{1}\right), \cdots, X_{n} k_{n}\left(i x_{n}-y_{n}\right)\right) \\
& =\left(k_{1}\left(i x_{1}-y_{1}\right), \cdots, k_{j}\left(i x_{j}-y_{j}\right), \cdots, k_{n}\left(i x_{n}-y_{n}\right)\right) .
\end{aligned}
$$

Assim, os campos definidos pela ação infinitesimal associada a $\psi$ são

$$
X_{j}^{\sharp}=k_{j}\left(x_{j} \frac{\partial}{\partial y_{j}}-y_{j} \frac{\partial}{\partial x_{j}}\right), j=i, \cdots, n .
$$

\section{- Passo 2: Cálculo da função hamiltoniana $\mu^{X}$ para o campo vetorial} $X^{\sharp}$

$$
d \mu^{X_{j}}=i_{X_{j}^{\sharp}} \omega=i_{k_{j}\left(x_{j} \frac{\partial}{\partial y_{j}}-y_{j} \frac{\partial}{\partial x_{j}}\right)} \sum_{j=i}^{n} d x_{j} \wedge d y_{j}=-k_{j}\left(x_{j} d x_{j}+y_{j} d y_{j}\right)
$$

portanto,

$$
\mu^{X_{j}}(z)=-\frac{k_{j}}{2}\left(x_{j}^{2}+y_{j}^{2}\right)+C_{j}=-\frac{k_{j}}{2}\left|z_{j}\right|^{2}+C_{j} .
$$

\section{- Passo 3: Cálculo da aplicação momento}

Como $\mu^{X_{j}}(z)=\langle\mu(z), X\rangle$ e $X=\left(X_{1}, \cdots, X_{n}\right)$, observemos que a aplicação momento é definida por

$$
\begin{aligned}
\mu: \mathbb{C}^{n} & \longrightarrow \mathbb{R}^{n} \\
z & \longmapsto\left(\mu^{X_{1}}(z), \cdots, \mu^{X_{n}}(z)\right)
\end{aligned}
$$

então

$$
\mu\left(z_{1}, \cdots, z_{n}\right)=\left(-\frac{1}{2} k_{1}\left|z_{1}\right|^{2}, \cdots,-\frac{1}{2} k_{n}\left|z_{n}\right|^{2}\right)+C
$$

III) Seja $\mathbb{S}^{1}$ atuando sobre $\mathbb{C}^{n}$ pela ação

$$
\begin{aligned}
\psi_{\xi}: \mathbb{S}^{1} \times \mathbb{C}^{n} & \longrightarrow \mathbb{C}^{n} \\
(\xi, z) & \longmapsto\left(\xi^{k} z_{1}, \cdots, \xi^{k} z_{n}\right)
\end{aligned}
$$

com $k \in \mathbb{Z}$ fixo. Semelhante aos itens anteriores, temos:

${ }^{c}$ Aqui usaremos o fato de que $X$ é base de $\mathbb{R}^{n}$, portanto, $X_{j}=e_{j}, \forall j=1, \cdots, n$. 
- Passo 1: Cálculo do campo fundamental e do campo vetorial $X^{\sharp}$

$$
\begin{aligned}
& \left.\frac{d}{d t} \psi(\exp (t X), z)\right|_{t=0}=k\left(i x_{1}-y_{1}, \cdots, i x_{n}-y_{n}\right)=k(i x-y), x, y \in \mathbb{R}^{n} \\
& \Rightarrow X^{\sharp}=k \sum_{j=1}^{n}\left(-y_{j} \frac{\partial}{\partial x_{j}}+x_{j} \frac{\partial}{\partial y_{j}}\right) .
\end{aligned}
$$

- Passo 2: Cálculo da função hamiltoniana $\mu^{X}$ para o campo vetorial $X^{\sharp}$

$$
\begin{aligned}
d \mu^{X}=i_{X^{\sharp} \omega} & =i_{k \sum_{j=1}^{n}\left(x_{j} \frac{\partial}{\partial y_{j}}-y_{j} \frac{\partial}{\partial x_{j}}\right)} \sum_{j=i}^{n} d x_{j} \wedge d y_{j}=-k \sum_{j=1}^{n}\left(x_{j} d x_{j}+y_{j} d y_{j}\right) \\
\Rightarrow \mu^{X}(z) & =-\frac{k}{2} \sum_{j=1}^{n}\left(x_{j}^{2}+y_{j}^{2}\right)+C=-\frac{k}{2} \sum_{j=1}^{n}\left|z_{j}\right|^{2}+C=-\frac{k}{2}\left(\left|z_{1}\right|^{2}+\cdots+\left|z_{n}\right|^{2}\right)+C \\
& =-\frac{k}{2}|z|^{2}+C .
\end{aligned}
$$

- Passo 3: Cálculo da aplicação momento

$\mu^{X}(z)=\langle\mu(z), X\rangle$ e $X=1 \Rightarrow \mu\left(z_{1}, \cdots, z_{n}\right)=\mu^{X}(z)=-\frac{k}{2}|z|^{2}+C$.

Nota: Observe que o exemplo acima não é o de uma ação tórica, pois a dimensão do grupo de Lie $\mathbb{S}^{1}$ não é a metade da dimensão da variedade $\mathbb{C}^{n}$.

IV) Seja $\left(\mathbb{S}^{2}, \omega=d \theta \wedge d h\right)$ e considere a ação do círculo $\mathbb{S}^{1}$ :

$$
\begin{aligned}
\psi: \mathbb{S}^{1} \times \mathbb{S}^{2} & \longmapsto \mathbb{S}^{2} \\
\left(e^{i t},(\theta, h)\right) & \longmapsto(\theta+t, h)
\end{aligned}
$$

- Passo 1: Cálculo do campo fundamental e do campo vetorial $X^{\sharp}$ Como Lie $\left(\mathbb{S}^{1}\right)=\mathbb{R}$, então $\exp (X)=e^{2 \pi i X} \in \mathbb{S}^{1}, X$ base de $\mathbb{R}$. Assim:

$$
\begin{aligned}
& \left.\frac{d}{d t} \psi\left(e^{i t X},(\theta, h)\right)\right|_{t=0}=\left.\frac{d}{d t}(\theta+t X, h)\right|_{t=0} \\
& =(X, 0)=(1,0) \\
& \Rightarrow X^{\sharp}=1 \frac{\partial}{\partial \theta}+0 \frac{\partial}{\partial h}=\frac{\partial}{\partial \theta} .
\end{aligned}
$$




\section{- Passo 2: Cálculo da função hamiltoniana $\mu^{X}$ para o campo vetorial $X^{\sharp}$ \\ $d \mu^{X}=i_{X^{\sharp} \omega}=i_{\frac{d}{d \theta}}(d \theta \wedge d h)=d h \Rightarrow \mu^{X}(\theta, h)=h$.}

\section{- Passo 3: Cálculo da aplicação momento}

$\mu^{X}(\theta, h)=\langle\mu(\theta, h), X\rangle$. Como $X=1$, então $\mu(\theta, h)=h$.

Seja $f: A \longrightarrow B$ uma aplicação linear entre espaços vetoriais e seja $f^{*}: B^{*} \longrightarrow A^{*}$ a sua aplicação dual (ou adjunta), i.e, a aplicação

$$
\left\langle f^{*}(b), a\right\rangle=\langle b, f(a)\rangle, a \in A, b \in B^{*} .
$$

Consideremos o espaço $\mathbb{C}^{d}$ equipado com a ação do toro $\mathbb{T}^{d}$ :

$$
\begin{aligned}
& \mathbb{T}^{d} \times \mathbb{C}^{d} \quad \longrightarrow \mathbb{C}^{d} \\
& \left(\left(e^{i t_{1}}, \cdots, e^{i t_{d}}\right),\left(z_{1}, \cdots, z_{d}\right)\right) \longmapsto\left(e^{i t_{1}} z_{1}, \cdots, e^{i t_{d}} z_{d}\right)
\end{aligned}
$$

tal ação é hamiltoniana com aplicação momento

$$
\mu\left(z_{1}, \cdots, z_{d}\right)=-\frac{1}{2}\left(\left|z_{1}\right|^{2}, \cdots,\left|z_{d}\right|^{2}\right)+\left(\lambda_{1}, \cdots, \lambda_{d}\right)
$$

e o subtoro $N=\mathbb{T}^{n-d}$ atua por restrição sobre $\mathbb{C}^{d}$ com aplicação momento

$$
\mu_{N}: i^{*} \circ \mu: \mathbb{C}^{d} \longrightarrow \mathfrak{n}^{*}
$$

para $\mathfrak{n}=\operatorname{Lie}(N)$

Lema 4.13 Seja $(M, \omega)$ uma variedade simplética e $N$ um subgrupo do grupo de Lie compacto $G$, com suas respectivas álgebras de Lie $\mathfrak{n}$ e $\mathfrak{g}$. Seja $i: \mathfrak{n} \hookrightarrow \mathfrak{g}$ a inclusão e $i^{*}: \mathfrak{g}^{*} \longrightarrow \mathfrak{n}^{*}$ sua aplicação dual.

Suponha que $G$ atue em $M$ de forma hamiltoniana com a aplicação momento $\mu: M \longrightarrow \mathfrak{g}^{*}$. Então, a restrição da ação de $N$ em $M$ é também hamiltoniana com aplicação momento dada pela composição $i^{*} \circ \mu: M \longrightarrow \mathfrak{n}^{*}$.

Prova. Seja $X \in \mathfrak{n}$. Então

$$
d\left(\left(i^{*} \circ \mu\right)^{X}\right)=d\left(\mu^{i(X)}\right)=\iota_{i(X)} \omega=\iota_{X} \omega
$$

sendo $\iota_{i(X)} \omega$ a contração da forma $\omega$ pelo campo $i(X) \in \mathfrak{g}$. 
A equivariância de $i^{*} \circ \mu$ vem do fato de $\mu$ ser equivariante e da igualdade $i=d_{e} f$, sendo $f: N \longrightarrow G$. Portanto, $N$ atua de forma hamiltoniana em $M$ com aplicação momento $i^{*} \circ \mu$.

Exemplo

Consideremos $\mathbb{C}^{d} \simeq \mathbb{R}^{2 d}$ munido com a forma simplética canônica

$$
\omega_{0}=\sum_{j=1}^{d} d x_{j} \wedge d y_{j} .
$$

Seja $\psi$ a ação tórica hamiltoniana

$$
\begin{aligned}
& \mathbb{T}^{d} \times \mathbb{C}^{d} \quad \longrightarrow \mathbb{C}^{d} \\
& \left(\left(e^{i t_{1}}, \cdots, e^{i t_{d}}\right),\left(z_{1}, \cdots, z_{d}\right)\right) \longmapsto\left(e^{i t_{1}} z_{1}, \cdots, e^{i t_{d}} z_{d}\right) .
\end{aligned}
$$

Vimos, em 4.1, que a aplicação momento para esta ação é

$$
\begin{aligned}
\mu: \quad \mathbb{C}^{d} & \longrightarrow \mathbb{R}^{d} \\
\left(z_{1}, \cdots, z_{d}\right) & \longmapsto-\frac{1}{2}\left(\left|z_{1}\right|^{2}, \cdots,\left|z_{d}\right|^{2}\right)+\left(\lambda_{1}, \cdots, \lambda_{d}\right)
\end{aligned}
$$

consideremos $N=\mathbb{S}^{1} \subseteq \mathbb{T}^{d}$, que atua por restrição em $\mathbb{C}^{d}$ da forma

$$
\begin{aligned}
\mathbb{S}^{1} \times \mathbb{C}^{d} & \longrightarrow \mathbb{C}^{d} \\
\left(e^{i \theta},\left(z_{1} \cdots, z_{d}\right)\right) & \longmapsto\left(z_{1} \cdots, e^{i \theta} z_{j}, \cdots, z_{d}\right)
\end{aligned}
$$

para algum $j=1, \cdots, d$, i.e., a rotação em torno do eixo $z_{j}$. Nesse caso, a ação de $N$ é hamiltoniana com aplicação momento

$$
\begin{aligned}
& \mu_{N}:=i^{*} \circ \mu: \quad \mathbb{C}^{d} \quad \longrightarrow \mathfrak{n}^{*} \simeq \mathbb{R} \\
& \left(z_{1}, \cdots, z_{n}\right) \longmapsto-\frac{1}{2}\left|z_{j}\right|^{2}+\lambda_{j} .
\end{aligned}
$$

Teorema 4.14 (Convexidade- Atiyah, [13]; Guillemin-Sternberg, [14]) Seja $(M, \omega)$ uma variedade simplética compacta e conexa e seja $\psi: \mathbb{T}^{n} \times M \longrightarrow M$ uma ação tórica hamiltoniana com aplicação momento $\mu: M \longrightarrow \mathbb{R}^{n}$. Então:

(i) $\mu^{-1}(c)$ é um conjunto conexo, para todo $c \in \mathbb{R}^{n}$.

(ii) A imagem de $\mu(M)$ é o casco convexo ${ }^{d}$ em $\mathbb{R}^{n}$, gerado pelas imagens dos pontos fixos da ação tórica.

${ }^{d} \mathrm{O}$ casco convexo de um conjunto de pontos no plano é o menor polígono convexo que os envolve, isto é, todos os pontos do conjunto devem estar dentro do polígono ou sobre a sua borda. 
Nota: A imagem é chamada de polítopo momento.

Vejamos mais alguns exemplos:

V) Vimos, no exemplo IV, que a ação de $\mathbb{S}^{1}$ na esfera $\mathbb{S}^{2}$ possui aplicação momento $\mu(\theta, h)=h$. Neste caso, os pontos fixos são da forma

$$
\left(\mathbb{S}^{2}\right)^{\mathbb{S}^{1}}=\left\{p \in \mathbb{S}^{2} \mid t \cdot p=p\right\}=\{N\} \cup\{S\}, \forall t \in \mathbb{S}^{1},
$$

onde $N=(0,0,1)$ e $S=(0,0,-1)$. Portanto

$$
\Delta=\mu\left(\left(\mathbb{S}^{2}\right)^{\mathbb{S}^{1}}\right)=[-1,1]
$$

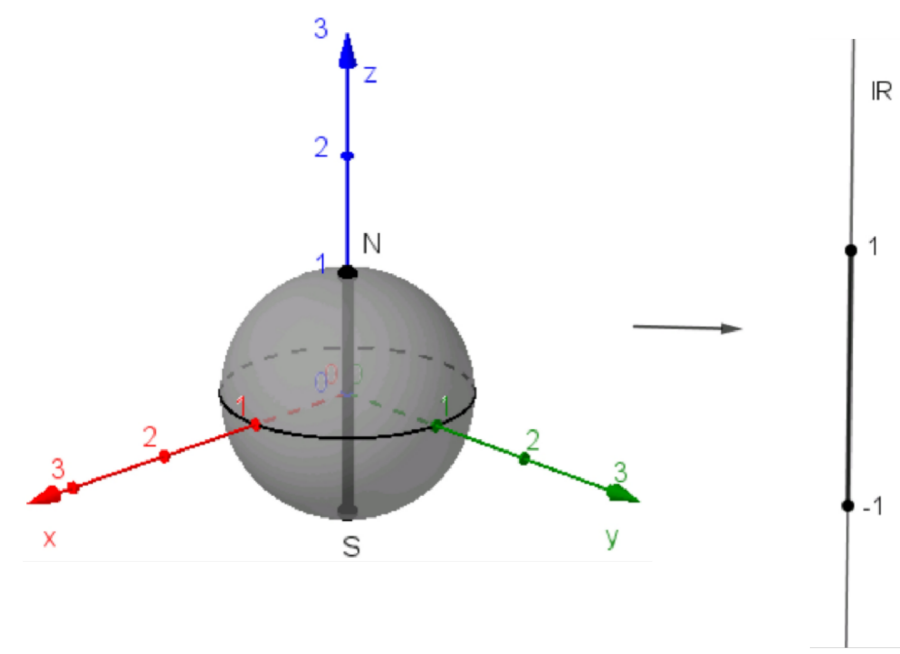

VI) Como último exemplo, citaremos a ação de $\mathbb{T}^{3}$ em $\mathbb{C P}^{3}$ definida como

$$
\begin{aligned}
\mathbb{T}^{3} \times \mathbb{C P}^{3} & \longmapsto \mathbb{C P}^{3} \\
\left(\left(e^{i t_{1}}, e^{i t_{2}}, e^{i t_{3}}\right),\left[z_{0}: z_{1}: z_{2}: z_{3}\right]\right) & \longmapsto\left[z_{0}: e^{i t_{1}} z_{1}: e^{i t_{2}} z_{2}: e^{i t_{3}} z_{3}\right]
\end{aligned}
$$

com a respectiva aplicação momento

$$
\begin{aligned}
\mu: \mathbb{C P}^{3} & \longrightarrow \mathbb{R}^{3} \\
{\left[z_{0}: z_{1}: z_{2}: z_{3}\right] } & \longmapsto-\frac{1}{2} \frac{\left(\left|z_{1}\right|^{2},\left|z_{2}\right|^{2},\left|z_{3}\right|^{2}\right)}{\left|z_{0}\right|^{2}+\left|z_{1}\right|^{2}+\left|z_{2}\right|^{2}+\left|z_{3}\right|^{2}}
\end{aligned}
$$


Os pontos fixos são mapeados da seguinte forma:

$$
\begin{aligned}
& {[1: 0: 0: 0]} \\
& {[0: 1: 0: 0]} \\
& {[0: 0(0,0,0)} \\
& {[0: 1: 0]} \\
& {[0: 0: 0: 1]}
\end{aligned}
$$

portanto, o polítopo é um simplexo em $\mathbb{R}^{3}$ cujos vértices são os pontos acima. Nesta dimensão, o simplexo é um tetraedro.

Definição 4.15 Seja $G$ um grupo de Lie e $M$ uma variedade. Dizemos que a ação $G \times M \longrightarrow M$ é efetiva se cada elemento do grupo $g \neq e$ move ao menos um ponto de $p \in M$, i.e., $\bigcap_{p \in M} G_{p}=\{e\}$, onde $G_{p}=\{g \in G \mid g \cdot p=p\}$ é o estabilizador de $p$.

Proposição 4.16 Seja $\left(M, \omega, \mathbb{T}^{n}, \mu\right)$ um $\mathbb{T}^{n}$-espaço hamiltoniano compacto e conexo. Então, para algum $p \in M$, a órbita $\mathbb{T}^{n} \cdot p$ é uma subvariedade isotrópica de $M$.

Prova. Como a aplicação momento $\mu$ é $\mathbb{T}^{n}$-invariante, então $\mu$ leva $\xi \in \mathfrak{t}^{*}$, $\mathfrak{t}=\operatorname{Lie}\left(\mathbb{T}^{n}\right)$, na órbita $\mathbb{T}^{n} \cdot p$. Segue que o diferencial $d \mu_{p}: T_{p} M \longrightarrow T_{\xi} \mathfrak{t}^{*} \simeq \mathfrak{t}^{*}$ leva o subespaço $T_{p}\left(\mathbb{T}^{n} \cdot p\right)$ em 0 , ou seja,

$$
T_{p}\left(\mathbb{T}^{n} \cdot p\right) \subset \operatorname{ker}\left(d \mu_{p}\right)=\left(T_{p}\left(\mathbb{T}^{n} \cdot p\right)\right)^{\omega_{p}}
$$

Portanto, $\mathbb{T}^{n} \cdot p$ é uma subvariedade isotrópica de $M$.

Proposição 4.17 Seja $\left(M, \omega, \mathbb{T}^{n}, \mu\right)$ um $\mathbb{T}^{n}$-espaço hamiltoniano compacto e conexo. Se a $\mathbb{T}^{n}$-ação é efetiva, então $\operatorname{dim} M \geq 2 n$.

Prova. Escolha qualquer $p \in M$ de modo que a $\mathbb{T}^{n}$-ação seja livre, isto é, $\left(\mathbb{T}^{n}\right)_{p}=\{e\}$. Assim, a órbita $\mathbb{T}^{n} \cdot p$ é difeomórfica a $\frac{\mathbb{T}^{n}}{\left(\mathbb{T}^{n}\right)_{p}}=\mathbb{T}^{n}$ e, portanto, tem dimensão $n$. Porém, como $\mathbb{T}^{n} \cdot p$ é uma subvariedade isotrópica de $M$, então

$$
n=\operatorname{dim}\left(\mathbb{T}^{n} \cdot p\right) \leq \frac{1}{2} \operatorname{dim} M
$$

Definição 4.18 Uma variedade tórica simplética é uma variedade simplética $(M, \omega)$ compacta e conexa de dimensão $2 n$, equipada com uma ação hamiltoniana efetiva de um toro de dimensão $n$, com a escolha de uma aplicação momento $\mu: M \longrightarrow \mathbb{R}^{n}$ correspondente. 
Definição 4.19 Duas variedades tóricas simpléticas $\left(M_{1}, \omega_{1}, \mathbb{T}_{1}, \mu_{1}\right)$ e $\left(M_{2}, \omega_{2}, \mathbb{T}_{2}, \mu_{2}\right)$ são equivalentes se, e somente se, existe um isomorfismo $\lambda: \mathbb{T}_{1} \longrightarrow \mathbb{T}_{2}$ e um simplectomorfismo $\lambda$-equivariante ${ }^{e} \varphi: M_{1} \longrightarrow M_{2}$ tal que

$$
\mu_{1}=\mu_{2} \circ \varphi
$$

\section{Exemplo}

Sejam $\mathbb{S}_{1}^{2}$ com a estrutura simplética $\omega_{1}=d \theta \wedge d h, \mathbb{S}_{r}^{2}$ com a estrutura simplética $\omega_{2}=\frac{1}{r} d \theta \wedge d h$ e considere as ações $\psi_{1}: \mathbb{S}^{1} \curvearrowright \mathbb{S}_{1}^{2}$ e $\psi_{r}: \mathbb{S}^{1} \curvearrowright \mathbb{S}_{r}^{2}$ por rotação ao redor do eixo z. Vamos calcular o isomorfismo $\lambda: \mathbb{S}^{1} \longmapsto \mathbb{S}^{1}$ que torna as variedades tóricas simpléticas $\left(\mathbb{S}_{1}^{2}, \omega_{1}, \mathbb{T}^{1}, \mu_{1}\right)$ e $\left(\mathbb{S}_{r}^{2}, \omega_{2}, \mathbb{T}^{1}, \mu_{2}\right)$ equivalentes: primeiramente, temos que para a ação

$$
\begin{aligned}
\psi_{1}: \mathbb{S}^{1} \times \mathbb{S}_{1}^{2} & \longmapsto \mathbb{S}_{1}^{2} \\
\left(e^{i t},(\theta, h)\right) & \longmapsto(\theta+t, h)
\end{aligned}
$$

$\mu_{1}(\theta, h)=h$ é uma aplicação momento, como calculada no exemplo IV. Agora, calculemos a aplicação momento $\mu_{2}$ referente à ação

$$
\begin{aligned}
\psi_{2}: \mathbb{S}^{1} \times \mathbb{S}_{r}^{2} & \longmapsto \mathbb{S}_{r}^{2} \\
\left(e^{i t},(\theta, r h)\right) & \longmapsto(\theta+t, r h) .
\end{aligned}
$$

Assim, seja $X_{2}=1 \in \operatorname{Lie}\left(\mathbb{S}^{1}\right)$, temos

- $\left.\frac{d}{d t} \psi_{2}\left(\exp t X_{2},(\theta, r h)\right)\right|_{t=0}=\left.\frac{d}{d t}\left(\theta+t X_{2}, r h\right)\right|_{t=0}=\left(X_{2}, 0\right)=(1,0)$.

Analogamente, $X_{2}^{\sharp}=\frac{\partial}{\partial \theta}$

- $i_{X_{2}^{\sharp} \omega_{2}}=i_{\frac{\partial}{\partial \theta}}\left(\frac{1}{r} d \theta \wedge d h\right)=\frac{1}{r} d h \Rightarrow \mu_{2}^{X_{2}}(\theta, r h)=\frac{h}{r}+C$

- $\mu_{2}^{X_{2}}(\theta, r h)=\left\langle\mu_{2}(\theta, r h), X_{2}\right\rangle \Rightarrow \mu_{2}(\theta, r h)=\frac{h}{r}+C$.

Portanto, $\left(\mathbb{S}_{1}^{2}, \omega_{1}, \mathbb{S}^{1}, \mu_{1}\right)$ é equivalente a $\left(\mathbb{S}_{r}^{2}, \frac{1}{r} \omega_{1}, \mathbb{S}^{1}, \frac{1}{r} \mu_{1}\right)$ se, e somente se, existe isomorfismo $\lambda: \mathbb{S}^{1} \longmapsto \mathbb{S}^{1}$ tal que o simplectomorfismo $\varphi: \mathbb{S}_{1}^{2} \longmapsto \mathbb{S}_{r}^{2}$, que associa $p=(\theta, h)$ a $\varphi(p)=(\theta, r h)$, é $\lambda$-equivariante. Isto é,

${ }^{e} \varphi$ é $\lambda$-equivariante $\Leftrightarrow \varphi\left(t \diamond m_{1}\right)=\lambda(t) \star \varphi\left(m_{1}\right), \forall m_{1} \in M_{1}$ e $t \in \mathbb{T}_{1}$, sendo $\diamond:=$ ação de $\mathbb{T}_{1}$ sobre $M_{1}$ e $\star$ : ação de $\mathbb{T}_{2}$ sobre $M_{2}$. 
- se $\varphi$ é um simplectomorfismo: de fato, temos

$$
\begin{aligned}
\left(\varphi^{*} \omega_{2}\right)_{p}\left(X_{1}, X_{2}\right) & =\left(\omega_{2}\right)_{\varphi(p)}\left(d_{p} \varphi\left(X_{1}\right), d_{p} \varphi\left(X_{2}\right)\right) \\
& =\frac{1}{r} d \theta \wedge d(r h) \\
& =d \theta \wedge d h=\omega_{1},
\end{aligned}
$$

- se $\varphi$ é $\lambda$-equivariante:

$$
\begin{aligned}
\varphi(t \cdot(\theta, h))=\lambda(t) \cdot \varphi(\theta, h) & \Leftrightarrow \varphi(\theta+t, h)=(\theta+\lambda(t), r h) \\
& \Leftrightarrow(\theta+t, r h)=(\theta+\lambda(t), r h) \\
& \Leftrightarrow \lambda(t)=t .
\end{aligned}
$$

Logo, as variedades simpléticas são equivalentes para o isomorfismo calculado por

$$
\begin{aligned}
\lambda: \mathbb{S}^{1} & \longmapsto \mathbb{S}^{1} \\
t & \longmapsto t .
\end{aligned}
$$




\section{5}

\section{Teorema de Delzant}

Neste capítulo veremos o conceito de polítopo necessário para enunciar um importante teorema, o Teorema de Delzant, que classifica as variedades tóricas simpléticas.

Definição 5.1 Um poliedro convexo é um subconjunto de $\mathbb{R}^{n}$ formado pela interseção de um número finito de semi-espaços afins.

Definição 5.2 Um polítopo em $\mathbb{R}^{n}$ é o casco convexo de um número finito de pontos em $\mathbb{R}^{n}$, isto é, um poliedro convexo delimitado.

Temos que $H \subseteq \mathbb{R}^{n}$ é um hiperplano se $H:=\left\{x \in \mathbb{R}^{n} \mid\langle x, v\rangle=a\right\}$, sendo $v \in \mathbb{R}^{n}$ vetor não nulo e $a \in \mathbb{R}$. $H$ é um hiperplano suporte de $X \in \mathbb{R}^{n}$ se $d(X, H)=\inf \{\|x-h\| \mid x \in X, h \in H\}=0$.

Definição 5.3 Seja $\triangle \subseteq \mathbb{R}^{n}$ um polítopo. Dizemos que o subconjunto $F=\triangle \cap H$ de $\triangle$, sendo $H$ o hiperplano suporte de $F$, é:

- vértice de $\triangle:$ Se $\operatorname{dim}(F)=0$,

- aresta de $\triangle$ : Se $\operatorname{dim}(F)=1$,

- faceta de $\triangle: \operatorname{Se} \operatorname{dim}(F)=d-1$ quando $\operatorname{dim}(\triangle)=d$.

Definição 5.4 Um polítopo de Delzant $\triangle$ é um polítopo em $\left(\mathbb{R}^{n}\right)^{*}$ que satisfaz:

(i) simplicidade: em cada vértice se encontram exatamente $n$ arestas,

(ii) racionalidade: as arestas que se encontram em cada vértice $p$ devem estar contidas entre pontos reticulares ${ }^{a}$, isto é, devem ser da forma $p+t u_{i}$, onde $u_{i} \in \mathbb{Z}^{n}$ para $t \geq 0$,

(iii) suavidade: em cada $p$, o correspondente $u_{1}, \cdots, u_{n}$ forma uma $\mathbb{Z}$-base de $\mathbb{Z}^{n}$.

${ }^{a}$ Pontos reticulares são aqueles que pertencem a uma rede diagonal em $\mathbb{R}^{n}$, que por sua vez é um subgrupo de $\mathbb{R}^{n}$ isomorfo a $\mathbb{Z}^{n}$ e que gera o espaço vetorial real $\mathbb{R}^{n}$. 
Exemplos de polítopos de Delzant:

- Em $\mathbb{R}^{0}$ : 0-polítopo equivale a um ponto (vértice).

- Em $\mathbb{R}$ : 1-polítopo equivale a um segmento (aresta).

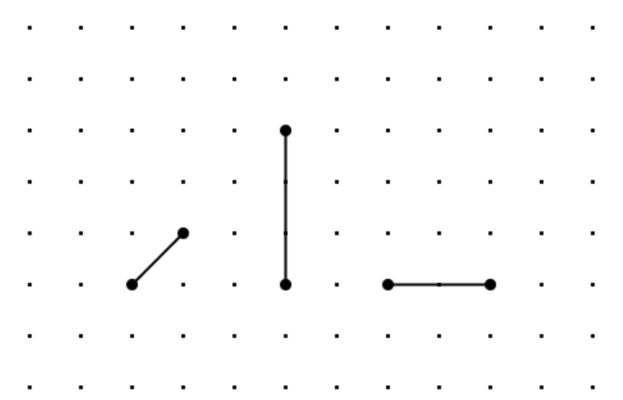

Figura 5.1: 1-polítopo de Delzant

A Figura 5.2 não representa um polítopo de Delzant porque o primeiro elemento fere a suavidade e o segundo elemento a racionalidade.

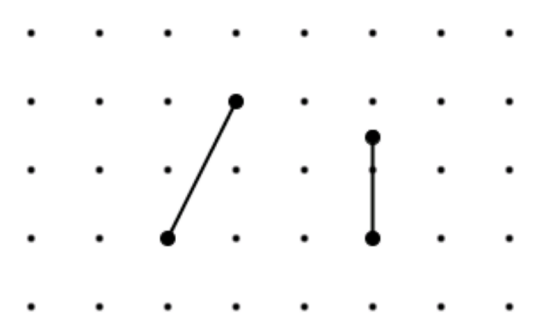

Figura 5.2: 1-polítopo não Delzant

- Em $\mathbb{R}^{2}$ : 2-polítopo

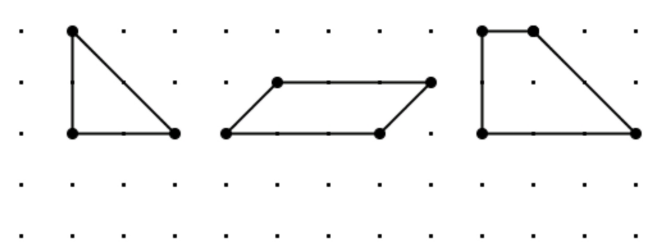

Figura 5.3: 2-polítopo de Delzant

Na Figura 5.4, os dois elementos falham na condição de suavidade. 


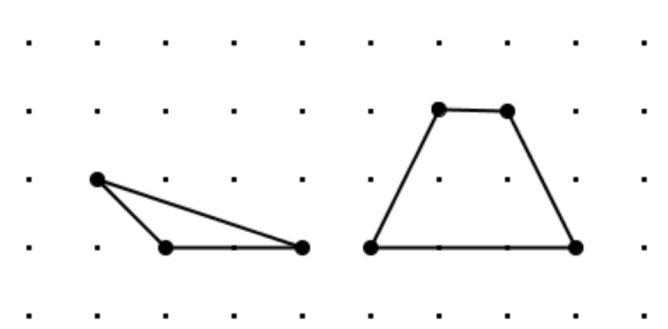

Figura 5.4: 2-polítopo não Delzant

- Em $\mathbb{R}^{3}$ : 3-polítopo

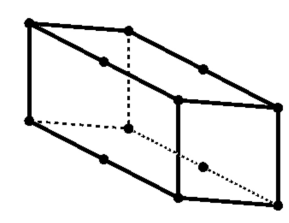

Figura 5.5: 3-polítopo de Delzant

Na Figura 5.6, o polítopo não é de Delzant porque não é simples, nem suave e nem racional.

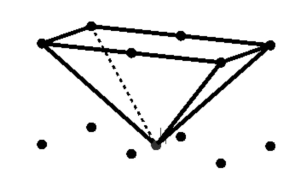

Figura 5.6: 3-polítopo não Delzant

Seja $\operatorname{Aff}_{\mathbb{Z}}\left(\mathbb{R}^{n}\right)$ o grupo afim que atua num ponto $x \in \mathbb{R}^{n}$ por $A x+\lambda$, com $A \in G L_{n}(\mathbb{Z})$ e $\lambda \in \mathbb{R}^{n}$. Note que se $\Delta$ é um polítopo de Delzant, também a sua imagem $g(\Delta)$ por $g \in \operatorname{Aff}_{\mathbb{Z}}\left(\mathbb{R}^{n}\right)$ é um polítopo de Delzant.

Teorema 5.5 (Delzant, [1]) Existe uma correspondência biunívoca entre variedades tóricas simpléticas equivalentes e polítopos de Delzant módulo a ação $\operatorname{Aff}\left(\mathbb{R}^{n}\right)$.

Essa correspondência se dá através da imagem da aplicação momento, ou seja, se $\left(M^{2 n}, \omega, \mathbb{T}^{n}, \mu\right)$ é uma variedade tórica simplética compacta, então $\mu(M)$ 
é seu polítopo de Delzant associado e vice-versa. Isto é, dado um polítopo de Delzant $\Delta$, é possível construir uma variedade tórica simplética $\left(M^{2 n}, \omega, \mathbb{T}^{n}, \mu\right)$ tal que $\mu(M)=\Delta$. Essa contrução será detalhada no Capítulo 7.

Podemos descrever um polítopo em $\left(\mathbb{R}^{n}\right)^{*}$ como a interseção de semiespaços:

$$
\triangle=\left\{x \in\left(\mathbb{R}^{n}\right)^{*} \mid\left\langle x, v_{i}\right\rangle \leq \lambda_{i}, i=1, \cdots, d\right\}, \lambda_{i} \in \mathbb{R} .
$$

Nesta dissertação, acompanhando [15], escolheremos os vetores $v_{i}$ apontando para fora.

Um vetor $v \in \mathbb{Z}^{n}$ é primitivo se não pode ser escrito da forma $v=k u, u \in \mathbb{Z}^{n}, k \in \mathbb{Z} \mathrm{e}|k|>1$.

Proposição 5.6 Seja $\Delta$ um polítopo de Delzant. Então os vetores $v_{i}$ são todos primitivos.

Prova. Suponha, por absurdo, que exista $i \in\{1, \cdots, n\}$ tal que $v_{i}$ não seja primitivo. Portanto, existe $k \in \mathbb{Z},|k|>1$ tal que $v_{i}=k v$, para $v \in \mathbb{Z}^{n}$.

Como o conjunto $v_{1}, \cdots, v_{n}$ forma uma base de $\mathbb{Z}^{n}$ sobre $\mathbb{Z}$, então $v$ pode ser escrito como uma combinação linear desses vetores, isto é existem $\alpha_{1}, \cdots, \alpha_{n} \in \mathbb{Z}$ tais que

$$
v=\alpha_{1} v_{1}+\cdots+\alpha_{n} v_{n}
$$

Multipliquemos ambos os lados por $k$, assim

$$
v_{i}=\alpha_{1} k v_{1}+\cdots+\alpha_{i} k v_{i}+\cdots+\alpha_{n} k v_{n}
$$

portanto, $\alpha_{i} k=1 \Leftrightarrow k= \pm 1$. O que é uma contradição, já que $|k|>1$.

Exemplo

$$
\begin{aligned}
\triangle & =\left\{\left(x_{1}, x_{2}\right) \in\left(\mathbb{R}^{2}\right)^{*} / x_{1} \geqslant 0, x_{1}+x_{2} \leqslant k\right\}, k \in \mathbb{Z} \\
& =\left\{\left(x_{1}, x_{2}\right) \in \mathbb{R}^{2} /\langle\left(x_{1}, x_{2}\right), \underbrace{(-1,0)}_{v_{1}}\rangle \leqslant 0,\langle\left(x_{1}, x_{2}\right), \underbrace{(0,1)}_{v_{2}}\rangle \leqslant 0,\langle\left(x_{1}, x_{2}\right), \underbrace{(1,1)}_{v_{3}}\rangle \leqslant k\right\} .
\end{aligned}
$$




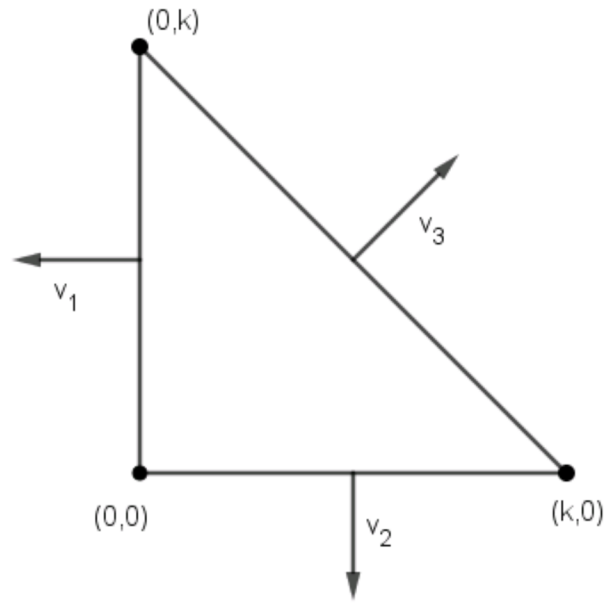

Figura 5.7: Vetores normais primitivos 


\section{6 \\ Redução Simplética}

A redução simplética é uma importante ferramenta utilizada para reduzir o total de simetrias de um sistema mecânico. A técnica consiste em considerar o quociente simplético, para tal, iremos utilizar o Teorema de Marsden-WeinsteinMeyer, que mostra que variedades simpléticas podem ser reduzidas por uma ação de um grupo de Lie, sob certas condições de regularidade e que tal quociente herda uma estrutura simplética.

\section{1}

\section{Espaço das Órbitas}

Vimos, no Capítulo 4, definições tais como órbita, grupo de isotropia de uma ação e sua classificação perante esses elementos (ação transitiva, livre e localmente livre). Neste breve capítulo iremos aprofundar sobre o espaço das órbitas, importante para construção da variedade tórica a partir de um polítopo de Delzant.

Seja a ação $\psi: G \times M \longrightarrow M$ entre um grupo de Lie $G$ e uma variedade $M$. Indicamos com $\sim$ a relação de equivalência de pertencer a uma mesma órbita, i.e., $p \sim q \Leftrightarrow p$ e $q$ estão na mesma órbita. Chamaremos de espaço das órbitas o quociente $\frac{M}{G}:=\frac{M}{\sim}$ e de projeção ponto-órbita a aplicação $\pi: M \longrightarrow \frac{M}{G}$. Além disso, temos que $\pi$ é chamada de quociente topológico quando temos ${ }^{a}$

$$
\pi^{-1}(U) \text { aberto em } M \Leftrightarrow U \text { aberto em } \frac{M}{G}, U \subseteq \frac{M}{G} .
$$

É importante notar que se o conjunto original é um espaço topológico, sempre será possível conferir ao quociente uma topologia para o qual a aplicação projeção seja contínua. Porém, no caso do espaço original ser uma variedade, o espaço quociente muitas vezes não é.

${ }^{a} \mathrm{~A}$ projeção $\pi: M \longrightarrow \frac{M}{G}$ é automaticamente contínua, pois a imagem inversa de aberto no conjunto $\frac{M}{G}$ é, por definição, aberta em $M$. 


\section{Exemplos}

1) Seja $G=\mathbb{C}^{*}$ atuando em $M=\mathbb{C}^{n}$ pela ação

$$
\begin{aligned}
\mathbb{C}^{*} \times \mathbb{C}^{n} & \longrightarrow \mathbb{C}^{n} \\
\left(\lambda,\left(z_{1}, \cdots, z_{n}\right)\right) & \longmapsto\left(\lambda z_{1}, \cdots, \lambda z_{n}\right)
\end{aligned}
$$

dado $z=\left(z_{1}, \cdots, z_{n}\right) \in \mathbb{C}^{n}$, temos:

órbita: $G \cdot z=\mathbb{C}^{*} z$, isto é, são linhas complexas menos a origem,

espaço das órbitas: $\frac{M}{G}=\frac{\mathbb{C}^{n}}{\mathbb{C}^{*}}=\mathbb{P}^{n-1} \sqcup\{*\}$.

Temos uma topologia quociente restrita ao espaço projetivo complexo (subvariedade do espaço das órbitas), logo, recuperamos a topologia usual de $\mathbb{P}^{n-1}$. Assim, a topologia em $\frac{M}{G}$ não é Hausdorff mas, ao remover o zero de $\mathbb{C}^{n}$, temos que $\mathbb{P}^{n-1}$ é um espaço das órbitas Hausdorff.

Nota: Além da identificação acima, também temos o espaço projetivo complexo associado pela ação do círculo como

$$
\mathbb{P}^{n-1}=\frac{\left(\mathbb{C}^{n}\right)^{*}}{\mathbb{C}^{*}}=\frac{\mathbb{S}^{2 n-1}}{\mathbb{S}^{1}}
$$

2) Análogo ao exemplo anterior, para $G=\mathbb{R}^{*}$ e $M=\mathbb{R}^{n}$, a ação

$$
\begin{aligned}
\mathbb{R}^{*} \times \mathbb{R}^{n} & \longrightarrow \mathbb{R}^{n} \\
\left(\lambda,\left(x_{1}, \cdots, x_{n}\right)\right) & \longmapsto\left(\lambda x_{1}, \cdots, \lambda x_{n}\right)
\end{aligned}
$$

possui, para $x=\left(x_{1}, \cdots, x_{n}\right) \in \mathbb{R}^{n}$ :

órbita: $G \cdot x=\mathbb{R}^{*} x$, ou seja, retas que passam pela origem (menos a origem) se $x \neq 0$ e um ponto quando $x=0$,

espaço das órbitas: $\mathbb{P}\left(\mathbb{R}^{n}\right) \cup\{*\}$.

Caso fosse considerada a mesma ação de $G$, mas em $\left(\mathbb{R}^{n}\right)^{*}$, o espaço das órbitas seria apenas o espaço projetivo real.

3) $G=\mathbb{R}$ atuando em $M=\mathbb{R}$ pela ação

$$
\begin{aligned}
\mathbb{R} \times \mathbb{R} & \longrightarrow \mathbb{R} \\
(t, a) & \longmapsto e^{t} a
\end{aligned}
$$

temos: 
órbitas: $G . a=\mathbb{R}^{+}, \mathbb{R}^{-}$e $\{0\}$, quando $a>0, a<0$ e $a=0$, respectivamente e

espaço das órbitas: $\frac{M}{G}=\frac{\mathbb{R}}{\sim}$, sendo a relação de equivalência definida por $a \sim b \Leftrightarrow a$ e $b$ pertencem à mesma órbita, isto é, se têm o mesmo sinal. Desta forma, o espaço das órbitas consiste em três pontos, cada um correspondente às órbitas semirreta positiva, semirreta negativa e origem. Portanto, a topologia quociente não é Hausdorff, já que não é possível termos vizinhanças disjuntas desses pontos.

\section{2}

\section{O Teorema de Marsden-Weinstein-Meyer}

Começamos relembrando, pela definição 3.14, que o anulador de $\mathfrak{g}_{p}$ é

$$
\mathfrak{g}_{p}^{0}=\left\{\xi \in \mathfrak{g}^{*} \mid\langle\xi, k\rangle=0, \forall k \in \mathfrak{g}_{p}\right\}
$$

Lema 6.1 Sejam $G$ um grupo de Lie e $(M, \omega)$ uma variedade simplética.

Seja $\psi: G \times M \longrightarrow M$ uma ação hamiltoniana e seja $\mu: M \longrightarrow \mathfrak{g}^{*}$ a aplicação momento, com $\mathfrak{g}=\operatorname{Lie}(G)$. Considere $\mathfrak{g}_{p}=\operatorname{Lie}\left(G_{p}\right)$. Então, para $d \mu_{p}: T_{p} M \longrightarrow \mathfrak{g}^{*}$, temos

i) $\operatorname{ker}\left(d \mu_{p}\right)=\left(T_{p}(G \cdot p)\right)^{\omega_{p}}$

ii) $\operatorname{im}\left(d \mu_{p}\right)=\mathfrak{g}_{p}^{0}$

Prova. Para algum $v \in T_{p} M$ e $\xi \in \mathfrak{g}$, temos $\omega_{p}\left(\left(X^{\xi}\right)_{p}, v\right)=\left\langle d \mu_{p}(v), \xi\right\rangle$, sendo $X^{\xi}$ o campo fundamental de $\xi$.

Como $\left(T_{p}(G \cdot p)\right)^{\omega_{p}}=\left\{v \in T_{p} M \mid \omega_{p}(v, w)=0, \forall w \in T_{p}(G \cdot p)\right\}$, disto segue que $\operatorname{ker}\left(d \mu_{p}\right)=\left(T_{p}(G \cdot p)\right)^{\omega_{p}}$.

A prova do segundo item resulta do Teorema do Núcleo e da Imagem e da proposição 3.16. Como

$$
\begin{gathered}
\operatorname{dim} \mathfrak{g}_{p}^{0}=\operatorname{dim} \mathfrak{g}-\operatorname{dim} \mathfrak{g}_{p} \\
\operatorname{dim}\left(T_{p}(G \cdot p)\right)^{\omega_{p}}=\operatorname{dim} T_{p} M-\left(\operatorname{dim} \mathfrak{g}-\operatorname{dim} \mathfrak{g}_{p}\right) .
\end{gathered}
$$

De fato,

$\operatorname{dim} \mathfrak{g}_{p}^{0}+\operatorname{dim}\left(T_{p}(G \cdot p)^{\omega_{p}}\right)=\operatorname{dim} T_{p} M=\operatorname{dim}\left(\operatorname{ker}\left(d \mu_{p}\right)\right)+\operatorname{dim}\left(\operatorname{im}\left(d \mu_{p}\right)\right)$. 
Devemos mostrar que $\operatorname{im}\left(d \mu_{p}\right) \subset \mathfrak{g}_{p}^{0}$ e que $\operatorname{ker}\left(d \mu_{p}\right) \subset T_{p}(G \cdot p)^{\omega_{p}}$. Como, $\forall \xi \in \mathfrak{g}, v \in T_{p} M$, temos $\omega_{p}\left(\left(X^{\xi}\right)_{p}, v\right)=\left\langle d \mu_{p}(v), \xi\right\rangle$, então

$$
v \in \operatorname{ker}\left(d \mu_{p}\right) \Rightarrow \omega_{p}\left(\left(X^{\xi}\right)_{p}, v\right)=0, \forall \xi \in \mathfrak{g}
$$

Assim, $\operatorname{ker}\left(d \mu_{p}\right) \subset T_{p}(G \cdot p)^{\omega_{p}}$. Também,

$$
X^{\xi}(p)=\left.\frac{d}{d t} \psi(\exp (t \xi), p)\right|_{t=0}=\left.\frac{d}{d t} p\right|_{t=0}=0, \forall \xi \in \mathfrak{g}_{p}
$$

logo, $\left\langle d \mu_{p}(v), \xi\right\rangle=0, \forall v \in T_{p} M \Rightarrow \operatorname{im}\left(d \mu_{p}\right) \subset \mathfrak{g}_{p}^{0}$.

Lema 6.2 Seja $(V, \omega)$ um espaço vetorial simplético e $Y \subset V$ um subespaço isotrópico de $V$, então $\omega$ induz uma forma simplética $\omega_{\text {red }}$ em $\frac{Y^{\omega}}{Y}$.

Prova. Considere $u, v \in Y^{\omega}$, então $[u],[v] \in \frac{Y^{\omega}}{Y}$ define

$$
\omega_{\text {red }}([u],[v])=\omega(u, v) .
$$

Assim, $\omega_{\text {red }}$ é fechada porque $\omega$ é fechada. Devemos mostrar que $\omega_{\text {red }}$ está bem definida e que é não degenerada. Para isso,

$$
u_{1} \in[u], v_{1} \in[v] \Rightarrow u_{1}=u+x, v_{1}=v+y, \forall x, y \in Y
$$

como $Y \subset Y^{\omega}$ porque $Y$ é isotrópico, temos

$$
\begin{aligned}
\omega\left(u_{1}, v_{1}\right) & =\omega(u+x, v+y) \\
& =\omega(u, v)+\omega(u, y)+\omega(x, v)+\omega(x, y) \\
& =\omega(u, v)
\end{aligned}
$$

observe que $\omega(u, y)=\omega(x, v)=0$, pois $u, v \in Y^{\omega}$ e $\omega(x, y)=0$ porque $x, y \in Y$ e $x, y \in[0]$. Assim,

$$
\omega_{\text {red }} \text { não degenerada } \Leftrightarrow \omega_{\text {red }}([u],[v])=0 \Rightarrow[v]=[0] .
$$

Dado $u \in Y^{\omega}$. Temos

$$
\omega_{\text {red }}([u],[v])=\omega(u, v)=0, \forall u \in Y^{\omega}
$$

portanto, $[v]=0$. 
Proposição 6.3 Uma $G$-ação é localmente livre em cada $p \in \mu^{-1}(0)$ se, e somente se, $d \mu_{p}$ é sobrejetivo, ou seja, se $p$ é ponto regular de $\mu: M \longrightarrow \mathfrak{g}^{*}$.

Prova. Temos que a ação é localmente livre em $p$ se, e somente se, $\mathfrak{g}_{p}=\{0\}$ e se, e somente se temos $\operatorname{im}\left(d \mu_{p}\right)=\mathfrak{g}^{*}$, ou seja, $d \mu_{p}$ é sobrejetivo.

Corolário 6.4 Se uma $G$-ação em $\mu^{-1}(0)$ é livre, então 0 é um valor regular de $\mu$ e, portanto, $\mu^{-1}(0)$ é uma subvariedade de $M$ de codimensão igual à dimensão de $G$.

Prova. Como $G$ age livremente sobre $\mu^{-1}(0)$, temos que $G_{p}=\{e\}$ para todo $p \in \mu^{-1}(0)$. Logo, $\mathfrak{g}_{p}=0$. Assim, a proposição 6.3 garante que $d \mu_{p}$ é sobrejetivo. Portanto, 0 é valor regular de $\mu$ e $\mu^{-1}(0)$ é subvariedade de $M$ com codimensão igual à dimensão de $G$.

Lema 6.5 Suponha que $G$ atua livremente em $\mu^{-1}(0)$ e que $0 \in \mathfrak{g}^{*}$ é um valor regular de $\mu$. Seja $p \in \mu^{-1}(0)$. Então $T_{p} \mu^{-1}(0)=T_{p}(G \cdot p)^{\omega}$, onde $G \cdot p \subset M$ é a órbita que passa por $p$. Em particular, $T_{p} \mu^{-1}(0)$ é um subespaço isotrópico de $T_{p} M$.

Prova. Temos que

$$
0 \text { valor regular } \Rightarrow T_{p} \mu^{-1}(0)=\operatorname{ker}\left(d \mu_{p}\right) \text {, para cada } p \in \mu^{-1}(0) \text {. }
$$

Isto significa que $T_{p} \mu^{-1}(0)=T_{p}(G \cdot p)^{\omega}$. Logo

$$
G \curvearrowright \mu^{-1}(0) \Rightarrow G \cdot p \in \mu^{-1}(0), \forall p \in \mu^{-1}(0) .
$$

Portanto, $T_{p}($ G.p $) \subset T_{p} \mu^{-1}(0)$.

Teorema 6.6 Se G é um grupo de Lie compacto e age livremente sobre $M$, então o espaço das órbitas $\frac{M}{G}$ é uma variedade e a aplicação $\pi: M \longrightarrow \frac{M}{G}$ é um G-fibrado principal.

Prova. ${ }^{b}$ Considere a ação

$$
\begin{aligned}
e v: G \times M & \longrightarrow M \\
(g, p) & \longmapsto g \cdot p
\end{aligned}
$$

se a ação é suave, ev é suave. Dado $p \in M$, considere a aplicação

$$
\begin{aligned}
e v_{p}: G & \longrightarrow M \\
g & \longmapsto g \cdot p
\end{aligned}
$$

${ }^{b}$ A demostração foi baseada em [15], p. 143. 
a imagem de $e v_{p}$ é uma $G$-órbita através de $p$ e ev $v_{p}$ é injetivo porque a ação de $G$ em $M$ é livre. Portanto, temos que para um subconjunto $A \subset M$ compacto e, portanto fechado, a sua imagem inversa $\left(e v_{p}\right)^{-1}(A)$ é também compacta (subconjunto fechado do grupo de Lie $G$ compacto).

Mostremos que ev $v_{p}$ é uma imersão. Para $X \in \mathfrak{g} \simeq T_{e} G$, temos

$$
d\left(e v_{p}\right)_{e}(X)=0 \Leftrightarrow X_{p}^{\sharp}=0 \Leftrightarrow X=0 .
$$

Logo, como a ação é livre, então $d\left(e v_{p}\right)_{e}$ é injetivo. Além disso, para $g \in G$, $X \in T_{g} G$, temos

$$
d\left(e v_{p}\right)_{g}(X)=0 \Leftrightarrow d\left(e v_{p} \circ R_{g}\right)_{e} \circ\left(d R_{g^{-1}}\right)_{g}(X)=0
$$

sendo $R_{g}: G \longrightarrow G$ a multiplicação pela direita por $g$. Assim, por $e v_{p} \circ R_{g}=e v_{g . p}$ possuir um diferencial injetivo em e e $\left(d R_{g^{-1}}\right)_{g}$ ser um isomorfismo, então $d\left(e v_{p}\right)_{g}$ é sempre injetivo.

Teorema 6.7 (Marsden-Weinstein [2], Meyer [3]) Seja $(M, \omega, G, \mu)$ um $G$ espaço hamiltoniano para um grupo de Lie $G$ compacto. Suponha que $G$ age livremente em $\mu^{-1}(0)$, então, se $i: \mu^{-1}(0) \hookrightarrow M$ é a inclusão, temos que:
i) o espaço das órbitas $M_{r e d}=\frac{\mu^{-1}(0)}{G}$ é uma variedade,
ii) a aplicação $\pi: \mu^{-1}(0) \longrightarrow M_{\text {red }}$ é um G-fibrado principal,
iii) existe uma forma simplética $\omega_{\text {red }}$ em $M_{\text {red }}$ tal que $i^{*} \omega=\pi^{*} \omega_{\text {red }}$.

Prova.

Temos, pelo corolário 6.4, que como $G$ atua livremente em $\mu^{-1}(0)$, então $0 \in \mathfrak{g}^{*}$ é valor regular de $\mu$ e, portanto, $\mu^{-1}(0)$ é uma subvariedade de $M$.

Além disso, pelo teorema 6.6, $M_{\text {red }}=\frac{\mu^{-1}(0)}{G}$ é uma variedade e a aplicação $\pi: \mu^{-1}(0) \longrightarrow M_{\text {red }}$ é um $G$-fibrado principal. Com isso, finalizamos a prova dos dois primeiros itens.

Para provar o terceiro item, temos pelo lema 6.5

$$
p \in \mu^{-1}(0) \Rightarrow T_{p}(G \cdot p)^{\omega_{p}}=\operatorname{ker}\left(d \mu_{p}\right)=T_{p} \mu^{-1}(0) .
$$

Assim, $T_{p}(G \cdot p)$ é isotrópico. O lema 6.2 garante que existe uma estrutura linear simplética em $T_{[p]} M_{\text {red }}$ induzida por $\omega_{p}$. 
Queremos mostrar que $\left(\omega_{\text {red }}\right)_{[p]}$ não depende da escolha do representante em $[p]$. Por isso, seja $q=g \cdot p$ com $g \in G$,

$$
\begin{array}{r}
d\left(e v_{g}\right)\left(T_{p} \mu^{-1}(0)\right)=T_{q} \mu^{-1}(0) \\
d\left(e v_{g}\right)\left(T_{p}(G \cdot p)_{p}\right)=T_{q}(G \cdot p)_{q} \\
\Rightarrow[u]=\left[d\left(e v_{g}\right)(u)\right] \in T_{[p]} M_{r e d}, \forall u \in T_{p} \mu^{-1}(0) .
\end{array}
$$

Logo, considerando a escolha de $p$ para a forma induzida $\omega_{\text {red }}$ e do ponto $q$ para a forma $\left(\omega_{\text {red }}\right)_{q}$, temos

$$
\begin{aligned}
\left(\omega_{\text {red }}\right)_{[p]}([v],[u]) & =\omega_{p}(u, v) \\
& =\omega_{[p]}\left(d\left(e v_{g}\right)(u), d\left(e v_{g}\right)(v)\right) \\
& =\left(\omega_{r e d}\right)_{[q]}\left(\left[d\left(e v_{g}\right)(u)\right],\left[d\left(e v_{g}\right)(v)\right]\right)
\end{aligned}
$$

Assim, se $\omega_{\text {red }}$ for fechada, então será uma forma simplética em M. De fato,

$$
\begin{aligned}
\pi^{*} d \omega_{\text {red }} & =d \pi^{*} \omega_{\text {red }} \\
& =d i^{*} \omega \\
& =i^{*} d \omega=0
\end{aligned}
$$

pela injetividade de $\pi^{*}$, segue que $d \omega_{\text {red }}=0$ ou seja, $\omega_{\text {red }}$ é fechada. Pelo lema $6.2, \omega_{\text {red }}$ é não degenerada e portanto, simplética.

A variedade simplética $\left(M_{r e d}, \omega_{r e d}\right)$ é chamada de quociente simplético ou de quociente de Marsden-Weinstein-Meyer da variedade $(M, \omega)$ com respeito à ação do grupo de Lie $G$.

Exemplos:

1) Recordemos a ação tórica do Grupo de Lie $G=\mathbb{S}^{1}$ sobre $\mathbb{C}$ do exemplo (I) da seção 4.3.

A aplicação momento é dada por $\mu(z)=-\frac{k}{2}|z|^{2}+C, z \in \mathbb{C}$ e $k \in \mathbb{Z}$ fixo, onde $C$ é uma constante que podemos escolher arbitrariamente. Escolhamos $C=\frac{k}{2}$. Assim, supondo que $G$ age livremente no conjunto de nível zero, temos

$$
\mu(z)=0 \Rightarrow|z|^{2}=1
$$


Portanto, $\mu^{-1}(0)=\left\{\left.z \in \mathbb{C}|| z\right|^{2}=1\right\}=\mathbb{S}^{1}$. A variedade reduzida, isto é, o espaço das órbitas da aplicação momento no nível zero é calculada por

$$
M_{r e d}=\frac{\mu^{-1}(0)}{G}=\frac{\mathbb{S}^{1}}{\mathbb{S}^{1}}=\{*\}
$$

com a estrutura simplética trivial.

2) Dada a ação do Grupo de Lie $G=\mathbb{S}^{1}$ sobre $\mathbb{C}^{n}$ do exemplo (III) da seção 4.3:

A aplicação momento é igual a $\mu(z)=-\frac{k}{2}|z|^{2}+C, z \in \mathbb{C}^{n}$ e $k \in \mathbb{Z}$ fixo, façamos novamente a escolha de $C=\frac{k}{2}$. Desta vez temos, para $\mathbb{S}^{1}$ agindo no conjunto de nível zero,

$$
\mu(z)=0 \Rightarrow|z|^{2}=1, z=\left(z_{1}, \cdots, z_{n}\right)
$$

Portanto, $\mu^{-1}(0)=\left\{\left.z \in \mathbb{C}^{n}|| z\right|^{2}=1\right\}=\mathbb{S}^{2 n-1}$. A variedade reduzida é calculada por

$$
M_{\text {red }}=\frac{\mu^{-1}(0)}{G}=\frac{\mathbb{S}^{2 n-1}}{\mathbb{S}^{1}}=\mathbb{P}^{n-1}
$$

conhecido como o espaço projetivo complexo, equipado com a forma simplética $\omega_{\text {red }}=\omega_{F S}$ chamada de forma Fubini-Study. ${ }^{c}$

${ }^{c}$ Temos que, para $M_{\text {red }}=\mathbb{P}^{1} \simeq \mathbb{S}^{2}, \omega_{F S}=\frac{1}{4} d \theta \wedge d h$. Para melhor aprofundar, consulte [16]. 


\section{7 \\ Construção de Delzant}

Em seu artigo [1], Thomas Delzant descreve como construir, dado um polítopo de Delzant $\Delta \subseteq\left(\mathbb{R}^{n}\right)^{*}$, uma variedade tórica simplética $\left(M, \omega, \mathbb{T}^{n}, \mu\right)$ tal que $\mu(M)=\Delta$. Neste capítulo descrevemos essa construção.

Dado um polítopo de Delzant, os passos seguintes levam à construção da variedade tórica simplética associada a ele:

Passo 1: Identificar os dados do polítopo: dimensão, facetas, vetores normais primitivos e descrever o polítopo por meio de inequações.

Passo 2: Temos que a construção descreve uma sequência exata ${ }^{a}$ entre grupos de Lie e suas respectivas álgebras de Lie:

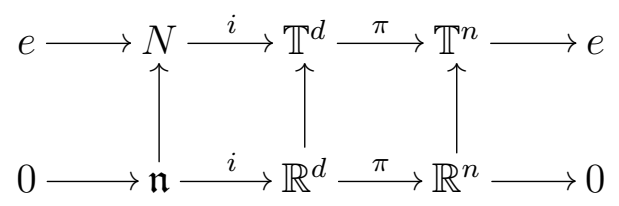

Este passo consiste em determinar $N$ e, posteriormente, a aplicação $i$.

Passo 3: Determinada $i$, chegamos à aplicação momento $\mu_{N}: i^{*} \circ \mu$ que, por sua vez, nos indica o conjunto de nível zero $Z=\mu_{N}^{-1}(0)$ e, finalmente, temos a variedade tórica simplética $M_{\Delta}$ associada ao polítopo de Delzant pelo quociente de Marsden-Weinstein-Meyer $Z / N$.

A construção faz uso da redução simplética, que prova a existência (ou sobrejetividade) do Teorema de Delzant

$$
\left[\left\{\left(M, \omega, \mathbb{T}^{d}, \mu\right)\right\}\right] \rightarrow[\{\text { polítopos de Delzant }\}] .
$$

Seja $\Delta$ um polítopo de Delzant com $d$ facetas. Podemos descrever $\Delta$ analiticamente, associando a cada semi-espaço que contém $\Delta$ um vetor não nulo $v_{i} \in \mathbb{Z}^{n}$, ortogonal (apontando para fora) à cada faceta e um número real $\lambda_{i}$ como

$$
\Delta=\left\{x \in\left(\mathbb{R}^{n}\right)^{*} \mid\left\langle x, v_{i}\right\rangle \leq \lambda_{i}, i=1, \cdots, d\right\}, d>n
$$

${ }^{a}$ Uma sequência de aplicações é exata se o núcleo de cada aplicação é igual à imagem da aplicação anterior. 
onde $\langle.,$.$\rangle é o produto interno euclidiano em \mathbb{R}^{n}$.

Seja $\left\{e_{1}, \cdots, e_{d}\right\}$ a base canônica de $\mathbb{R}^{d}$. Considere a aplicação

$$
\begin{aligned}
\pi: \mathbb{R}^{d} & \longrightarrow \mathbb{R}^{n} \\
e_{i} & \longmapsto v_{i}
\end{aligned}
$$

Lema 7.1 A aplicação $\pi$ é sobrejetora e mapeia $\mathbb{Z}^{d}$ em $\mathbb{Z}^{n}$, isto é, $\pi\left(\mathbb{Z}^{d}\right)=\mathbb{Z}^{n}$.

Prova. O conjunto $\left\{e_{1}, \cdots, e_{d}\right\}$ é base de $\mathbb{Z}^{d}$ sobre $\mathbb{Z}$. Iremos mostrar que o $\mathbb{Z}$ espaço gerado por $\left\{v_{1}, \cdots, v_{d}\right\}$ é $\mathbb{Z}^{n}$.

Pela simplicidade, em um vértice do polítopo, os vetores $u_{1}, \cdots, u_{n} \in\left(\mathbb{R}^{n}\right)^{*}$ formam uma base para $\left(\mathbb{Z}^{n}\right)^{*}$. Assumamos o conjunto desses vetores como base canônica, sem perda de generalidade. Disto, pela racionalidade, cada vetor normal primitivo $v_{i}$ que aponta para fora das facetas que se encontram no vértice é simétrico em relação ao vetor $u_{i}$ correspondente. Logo, pela suavidade, o conjunto deles forma uma base de $\mathbb{Z}^{n}$.

Como consequência do lema anterior, existe um homomorfismo sobrejetivo de grupos de Lie $\pi: \mathbb{T}^{d} \longrightarrow \mathbb{T}^{n}$ de forma que o seguinte diagrama comuta:

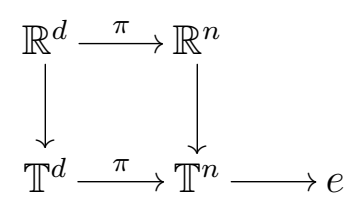

onde $\mathbb{T}^{d}=\frac{\mathbb{R}^{d}}{\mathbb{Z}^{d}}$ e $\mathbb{T}^{n}=\frac{\mathbb{R}^{n}}{\mathbb{Z}^{n}}$. O núcleo da sobrejeção $\pi$

$$
N:=\operatorname{ker} \pi=\left\{g \in \mathbb{T}^{d} \mid \pi(g)=e\right\}
$$

é um toro $(d-n)$-dimensional contido em $\mathbb{T}^{d}$, onde $i: N \hookrightarrow \mathbb{T}^{d}$ é a inclusão. Por $i$ ser injetiva, temos uma sequência exata de toros, que induz também uma sequência exata de suas respectivas álgebras de Lie.

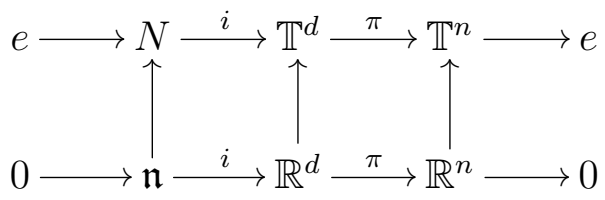

Desta forma, o dual da sequência das álgebras de Lie é a sequência exata

$$
0 \longrightarrow\left(\mathbb{R}^{n}\right)^{*} \stackrel{\pi^{*}}{\longrightarrow}\left(\mathbb{R}^{d}\right)^{*} \stackrel{i^{*}}{\longrightarrow} \mathfrak{n}^{*} \longrightarrow 0
$$


Seja $Z:=\mu_{N}^{-1}(0)=\left(i^{*} \circ \mu\right)^{-1}(0)$ o conjunto de nível zero.

Lema 7.2 Seja $\Delta=\left\{x \in\left(\mathbb{R}^{n}\right)^{*} \mid\left\langle x, v_{i}\right\rangle \leq \lambda_{i}, i=1, \cdots, d\right\}$ um polítopo de Delzant com $d$ facetas, $d>n$ e seja $\Delta^{\prime}$ sua imagem por $\pi^{*}$. Seja $\mu$ a aplicação momento da ação hamiltoniana do toro $\mathbb{T}^{d}$ na variedade $\mathbb{C}^{d}$ tal que $\mu: \mathbb{C}^{d} \longrightarrow\left(\mathfrak{t}^{d}\right)^{*} \simeq \mathbb{R}^{d}$, então

$$
\mu(Z)=\pi^{*}(\Delta)=\Delta^{\prime}
$$

Prova. Queremos provar que, dado $y \in\left(R^{d}\right)^{*}$, então

$$
y \in \Delta^{\prime} \Leftrightarrow y \in \mu(Z)
$$

Isso equivale a mostrar que $y \in \operatorname{im}(\mu)$ e $i^{*} y=0$. Pela sequência exata 7.1, essas condições equivalem às $\left\langle y, e_{i}\right\rangle \leqslant \lambda_{i}, i=1, \cdots, d$ e $y=\pi^{*}(x)$, para algum $x \in\left(\mathbb{R}^{n}\right)^{*}$.

A condição $y=\pi^{*}(x)$ implica que, para algum $x \in\left(\mathbb{R}^{n}\right)^{*}$ e $i \in\{1, \cdots, n\}$, temos

$$
\left\langle y, e_{i}\right\rangle=\left\langle\pi^{*}(x), e_{i}\right\rangle=\left\langle x, \pi\left(e_{i}\right)\right\rangle=\left\langle x, v_{i}\right\rangle
$$

Logo,

$$
\left\langle y, e_{i}\right\rangle \leqslant \lambda_{i} \Leftrightarrow\left\langle x, v_{i}\right\rangle \leqslant \lambda_{i} \Leftrightarrow x \in \Delta
$$

e, portanto, $y \in \mu(Z) \Leftrightarrow y \in \pi^{*}(\Delta)=\Delta^{\prime}$, ou seja, $\mu(Z)=\pi^{*}(\Delta)=\Delta^{\prime}$.

Lema 7.3 O conjunto $Z$ é compacto e $N$ atua livremente sobre $Z$.

Prova. Temos, pelo lema 7.2, que $Z \subseteq \mu^{-1}\left(\pi^{*}\left(\Delta^{\prime}\right)\right)$. Afirmamos que $Z$ é um subconjunto fechado de um conjunto compacto. De fato, $\mu^{-1}\left(\pi^{*}\left(\Delta^{\prime}\right)\right)$ é a imagem inversa do compacto $\pi^{*}\left(\Delta^{\prime}\right)$ pela aplicação momento $\mu$, que é própria ${ }^{b}$. Portanto, $Z$ é compacto.

Resta mostrar que $N$ age livremente sobre $Z$, isto é, que o estabilizador $N_{x}=\{e\}$ para $x \in Z$ arbitrário. Novamente pelo lema 7.2, existe $p \in \Delta$ tal que $\mu(x)=\pi^{*}(\langle p, \cdot\rangle)$. Disto, temos três casos:

(i) $p$ é vértice de $\Delta$.

\footnotetext{
${ }^{b}$ Uma aplicação $M_{1} \longrightarrow M_{2}$ é própria se para todo compacto $K \in M_{2}$, sua imagem inversa em $M_{1}$ é também compacta em $M_{1}$.
} 
Pela simplicidade de $\Delta$, existe $I=\left\{i_{1}, \cdots, i_{n}\right\}$ conjunto de índices tais que $p$ é caracterizado por equações $\left\langle p, v_{i}\right\rangle=\lambda_{i}, i \in I$. Isso equivale a

$$
\begin{gathered}
\left\langle p, v_{i}\right\rangle=\left\langle p, \pi\left(e_{i}\right)\right\rangle=\left\langle\pi^{*}(p), e_{i}\right\rangle=\left\langle\mu(x), e_{i}\right\rangle=\lambda_{i} \\
\Leftrightarrow-\frac{1}{2}\left|x_{i}\right|^{2}+\lambda_{i}=\lambda_{i} \\
\Leftrightarrow x_{i}=0 .
\end{gathered}
$$

Sem perda de generalidade, podemos assumir $I=\{1, \cdots, n\}$.

Assim, $x=\left(0, \cdots, 0, x_{n+1}, \cdots, x_{d}\right) ; x_{n+1} \neq 0, \cdots, x_{d} \neq 0$. Portanto

$$
\left(\mathbb{T}^{d}\right)_{x}=\left\{\left(t_{1}, \cdots, t_{n}, 1, \cdots, 1\right) \in \mathbb{T}^{d}\right\}
$$

Como a restrição $\pi:\left(\mathbb{R}^{d}\right)_{x} \longrightarrow \mathbb{R}^{n}$, que é uma $\mathbb{Z}$-base de $\mathbb{Z}^{n}$ pela suavidade, mapeia $e_{1}, \cdots, e_{n}$ em $v_{1}, \cdots, v_{n}$, segue que $\pi:\left(\mathbb{T}^{d}\right)_{x} \longrightarrow \mathbb{T}^{n}$ é bijetiva e $\operatorname{ker}\left(\left.\pi\right|_{\left(\mathbb{T}^{d}\right)_{x}}\right)=\{e\}$. Logo

$$
N \cap\left(\mathbb{T}^{d}\right)_{x}=\{e\}
$$

$E$, como $N_{x} \subseteq\left(\mathbb{T}^{d}\right)_{x}$, donde $N_{x} \subseteq N \cap\left(\mathbb{T}^{d}\right)_{x}=\{e\}$, então $N_{x}=\{e\}$.

(ii) $p$ é ponto interior de $\Delta$.

Neste caso, $p$ satisfaz as desigualdades

$$
\left\langle p, v_{i}\right\rangle<\lambda_{i}, \forall i=1, \cdots, d
$$

Análogo ao caso anterior, concluímos que $x_{i} \neq 0, \forall i=1, \cdots, d$, levando ao mesmo resultado $N_{x}=\{e\}$, então $N_{x} \subseteq\left(\mathbb{T}^{d}\right)_{x}=\{e\}$.

(iii) $p \in F, F=$ face (diferente dos vértices e de $\Delta$ ).

Também análogo aos casos anteriores.

Portanto, $N$ atua livremente sobre $Z$.

Lema 7.4 O conjunto de nível $Z=\left(i^{*} \circ \mu\right)^{-1}(0)$ é uma subvariedade de $\mathbb{C}^{d}$ com dimensão $d+n$. 
Prova. Vimos, pelo lema 7.3, que $Z$ é compacto. Alem disso, temos que a ação do toro $\mathbb{T}^{d}$ em $\mathbb{C}^{d}$ é livre, então sua aplicação momento $\mu: \mathbb{C}^{d} \longrightarrow\left(\mathbb{R}^{d}\right)^{*}$ é uma submersão. A aplicação inclusão $i: \mathfrak{n} \hookrightarrow \mathbb{R}^{d}$ é injetiva, logo, $i^{*}:\left(\mathbb{R}^{d}\right)^{*} \longrightarrow \mathfrak{n}^{*}$ é sobrejetiva.

Das afirmações acima, concluímos que $Z=\left(i^{*} \circ \mu\right)^{-1}(0)$ é uma subvariedade de $\mathbb{C}^{d}$ com dimensão real igual a

$$
\operatorname{dim}\left(\mathbb{C}^{d}\right)-\operatorname{dim}\left(\mathfrak{n}^{*}\right)=2 d-(d-n)=d+n
$$

Portanto, dado um polítopo de Delzant $\Delta$, é possível a construção da variedade tórica simplética reduzida $\left(M_{\Delta}, \omega_{\Delta}\right)$, donde

$$
M_{\Delta}=\frac{Z}{N}
$$

é uma variedade $2 n$-dimensional ${ }^{c}$ e $\omega_{\Delta}$ é a forma simplética reduzida determinada, segundo o teorema 6.7 da Redução Simplética de Marsden-Weinstein-Meyer, por

$$
i^{*} \omega=\pi^{*} \omega_{\Delta}
$$

onde $i: Z \hookrightarrow \mathbb{C}^{d}$ é a aplicação inclusão e $\pi: Z \longrightarrow M_{\Delta}$ é a aplicação quociente.

Lema 7.5 Seja $\sigma: \mathbb{T}^{n} \longrightarrow\left(\mathbb{T}^{d}\right)_{x}$ a aplicação inversa ${ }^{d}$ de $\pi:\left(\mathbb{T}^{d}\right)_{x} \longrightarrow \mathbb{T}^{n}$, para $x \in Z$. A ação residual

$$
\begin{aligned}
\psi: \mathbb{T}^{n} \times M_{\Delta} & \longrightarrow M_{\Delta} \\
(g, p(x)) & \longmapsto p(\sigma(g) \cdot x)
\end{aligned}
$$

é hamiltoniana com aplicação momento $\mu_{\Delta}$, cuja imagem $\mu_{\Delta}\left(M_{\Delta}\right)=\Delta$.

Prova. Seja $\mu: M \longrightarrow \mathbb{R}^{d}$, a aplicação momento da ação hamiltoniana do toro $\mathbb{T}^{d}$ na variedade $M$ então, pela $\mathbb{T}^{d}$-equivariância de $\mu$, temos $\mu(x)=\mu(\sigma(g) \cdot x)$ e, como $Z=\left(i^{*} \circ \mu\right)^{-1}(0)$, temos

$$
\left(i^{*} \circ \mu\right)^{-1}(\sigma(g) \cdot x)=\left(i^{*} \circ \mu\right)^{-1}(x)=0 .
$$

Logo, $Z$ é invariante pela ação de $\sigma\left(\mathbb{T}^{n}\right)$.

Observe que $\sigma: \mathbb{T}^{n} \longrightarrow\left(\mathbb{T}^{d}\right)_{x} \subseteq \mathbb{T}^{d}$. Temos sua aplicação dual das álgebras de Lie como $\sigma^{*}:\left(\mathbb{R}^{d}\right)^{*} \longrightarrow\left(\mathbb{R}^{n}\right)^{*}$. Disto, segue pela invariância de $\mu$ que o

${ }^{c} \operatorname{dim}_{\mathbb{R}} M_{\Delta}=\operatorname{dim} Z-\operatorname{dim} N=d+n-(d-n)=2 n$.

${ }^{d}$ Pelo lema $7.3,\left.\pi\right|_{\left(\mathbb{T}^{d}\right)_{x}}$ é uma bijeção. 
diagrama abaixo comuta:

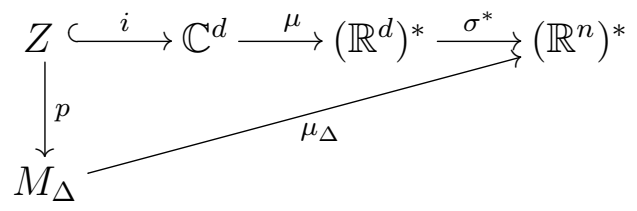

Portanto, a composta $\sigma^{*} \circ \mu \circ i$ é $N$-invariante. Logo, existe a aplicação momento $\mu_{\Delta}: M_{\Delta} \longrightarrow\left(\mathbb{R}^{n}\right)^{*}$ pela ação do toro $\mathbb{T}^{n}$ sobre $M_{\Delta}$. Logo, $\psi$ é uma ação tórica hamiltoniana com aplicação momento $\mu_{\Delta}$.

Agora, concluímos a demonstração afirmando que $\mu_{\Delta}\left(M_{\Delta}\right)=\Delta$. De fato,

$$
\begin{aligned}
\mu_{\Delta}\left(M_{\Delta}\right) & =\mu_{\Delta}(p(Z)) \\
& =\left(\sigma^{*} \circ \mu \circ i\right)(Z) \\
& =\sigma^{*}\left(\pi^{*}(\Delta)\right) \text { pelo lema } 7.2 \\
& =\left(\sigma^{*} \circ \pi^{*}\right)(\Delta) \\
& =(\pi \circ \sigma)^{*}(\Delta) \\
& =(I d)^{*}(\Delta)=(I d)(\Delta) \\
& =\Delta .
\end{aligned}
$$




\section{8 \\ Exemplos da Construção de Delzant}

Neste capítulo construiremos explicitamente alguns exemplos. Para isso, seguiremos os passos citados no início do capitulo anterior.

\section{1}

Segmento de Comprimento $L$

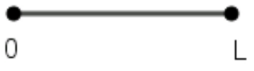

Passo 1 Descrição do polítopo

- Dimensão: $n=1$.

- Facetas: $d=2$ (aquelas que possuem dimensão igual à $n-1$, isto é, são os pontos 0 e $L$ ).

- $v=1$ (base vetorial canônica em $\mathbb{R}$ ).

$$
\begin{aligned}
\Delta & =\left\{x \in \mathbb{R}^{*} \mid 0 \leq x \leq L\right\}, L \in \mathbb{Z} \\
& =\left\{x \in \mathbb{R}^{*} \mid\langle x,-v\rangle \leq 0,\langle x, v\rangle \leq L\right\} \Rightarrow \lambda_{1}=0, \lambda_{2}=L .
\end{aligned}
$$

Passo 2 Determinar o núcleo $N$ e a aplicação inclusão $i$

. Projeção $\pi$ :

$$
\begin{aligned}
\pi: \mathbb{R}^{2} & \longrightarrow \mathbb{R} \\
e_{1} & \longmapsto-v \\
e_{2} & \longmapsto v
\end{aligned}
$$

Assim

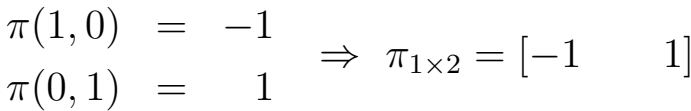

$$
\begin{aligned}
& \text { - Toro } N \text { : }
\end{aligned}
$$




$$
\begin{aligned}
N=\operatorname{ker} \pi \Rightarrow(a, b) \in N & \Leftrightarrow\left[\begin{array}{ll}
-1 & 1
\end{array}\right]\left[\begin{array}{ll}
a & b
\end{array}\right]^{T}=0 \\
& \Leftrightarrow-a+b=0 \\
& \Leftrightarrow(a, b)=(a, a)=a(1,1) .
\end{aligned}
$$

Disto, segue que $\operatorname{dim}(N)=1 \Rightarrow N=\mathbb{S}^{1}$. Temos, portanto, a sequência exata de toros e de suas respectivas álgebras de Lie:

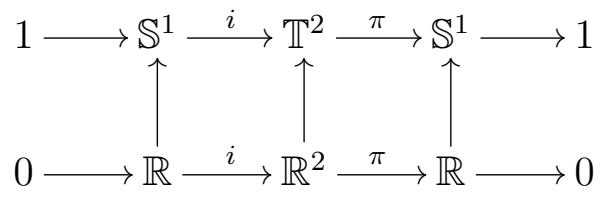

- Aplicação inclusão:

$$
\begin{aligned}
i: \mathbb{R} & \longrightarrow \mathbb{R}^{2} \\
a & \longmapsto(a, a)=a(1,1)
\end{aligned} \Rightarrow i_{2 \times 1}=\left[\begin{array}{ll}
1 & 1
\end{array}\right]^{T}
$$

- Dual da aplicação inclusão: Temos abaixo a sequência exata do dual das álgebras de Lie

$$
0 \longrightarrow \mathbb{R}^{*}=\mathbb{R} \stackrel{\pi^{*}}{\longrightarrow}\left(\mathbb{R}^{2}\right)^{*}=\mathbb{R}^{2} \stackrel{i^{*}}{\longrightarrow} \mathbb{R}^{*}=\mathbb{R} \longrightarrow 0
$$

Como $i^{*}=[i]^{T}=\left[\begin{array}{ll}1 & 1\end{array}\right]$, então

$$
\begin{aligned}
i^{*}: & \mathbb{R}^{2} \\
(a, b) & \longmapsto \mathbb{R} \\
& \longmapsto a+b
\end{aligned}
$$

Passo 3 Determinar a aplicação momento $\mu$, o conjunto de nível $Z$ e a variedade reduzida $M_{\Delta}$.

- Aplicação momento: Consideremos $\mathbb{C}^{2}$ com sua estrutura simplética canônica $\omega_{s t d}=\frac{i}{2} \sum_{i=1}^{2} d z_{i} \wedge d \bar{z}_{i}$ e com a ação diagonal de $\mathbb{T}^{2}$. Como calculado em 4.1, a aplicação momento pela ação diagonal de $\mathbb{T}^{2}$ em $\mathbb{C}^{2}$ é

$$
\begin{aligned}
& \mu: \mathbb{C}^{2} \longrightarrow \mathbb{R}^{2} \\
&\left(z_{1}, z_{2}\right) \longmapsto-\frac{1}{2}\left(\left|z_{1}\right|^{2},\left|z_{2}\right|^{2}\right)+\left(\lambda_{1}, \lambda_{2}\right) \\
& \Rightarrow \mu\left(z_{1}, z_{2}\right)=\left(-\frac{\left|z_{1}\right|^{2}}{2},-\frac{\left|z_{2}\right|^{2}}{2}+L\right) .
\end{aligned}
$$


Além disso, temos $\mu_{N}=i^{*} \circ \mu: \mathbb{C}^{2} \longrightarrow \mathbb{R}$ tal que

$$
\begin{aligned}
\mu_{N}\left(z_{1}, z_{2}\right) & =i^{*} \circ \mu\left(z_{1}, z_{2}\right) \\
& =i^{*}\left(-\frac{\left|z_{1}\right|^{2}}{2},-\frac{\left|z_{2}\right|^{2}}{2}+L\right) \\
& =-\frac{1}{2}\left(\left|z_{1}\right|^{2}+\left|z_{2}\right|^{2}\right)+L .
\end{aligned}
$$

- Conjunto de nível zero:

$Z=\mu_{N}^{-1}(0)$, então

$$
-\frac{1}{2}\left(\left|z_{1}\right|^{2}+\left|z_{2}\right|^{2}\right)+L=0 \Leftrightarrow L=\frac{1}{2}\left(\left|z_{1}\right|^{2}+\left|z_{2}\right|^{2}\right) \subset \mathbb{C}^{2} \simeq \mathbb{R}^{4}
$$

logo, $Z=\mathbb{S}_{\sqrt{2 L}}^{3}$ (observe aqui que $\operatorname{dim}_{\mathbb{R}} Z=d+n=3$ ).

- Variedade reduzida:

$$
M_{\Delta}=\frac{Z}{N}=\frac{\mathbb{S}^{3}}{\mathbb{S}^{1}} \simeq \mathbb{C P}^{1}
$$

Pelo Teorema 6.7, $\mathbb{C P}^{1}$ herda de $\left(\mathbb{C}^{2}, \omega_{s t d}\right)$ uma estrutura simplética definida pela equação

$$
i^{*} \omega_{s t d}=\pi^{*} \omega_{\text {red }}
$$

onde $i: Z \hookrightarrow \mathbb{C}^{2}$ é a inclusão e $\pi: Z \longrightarrow Z / N=\mathbb{C P}^{1}$ a projeção. Quando $L=\frac{1}{2}$, a forma simplética $\omega_{\text {red }}$ em $\mathbb{C P}^{1}$ é a forma Fubini-Study

$$
\omega_{F S}=\frac{i}{2} \frac{d z \wedge d \bar{z}}{\left(1+|z|^{2}\right)^{2}}
$$

\section{2}

\section{Triângulo Retângulo de Catetos $L$}

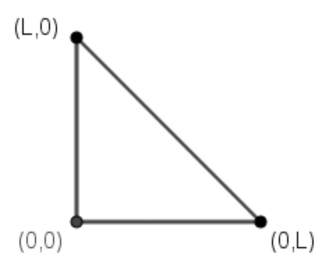

Passo 1 Descrição do polítopo 
- Dimensão: $n=2$.

- Facetas: $d=3$ (aquelas que possuem dimensão igual à $n-1$, isto é, são os lados do triângulo).

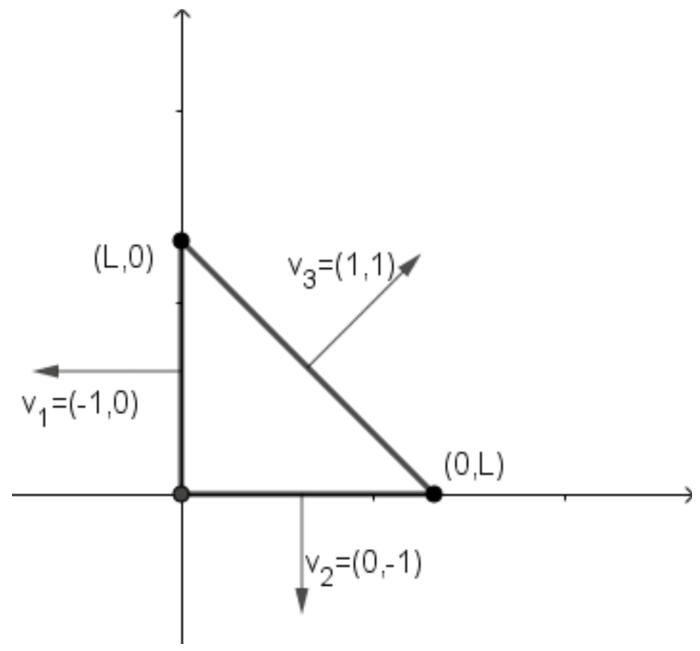

- Vetores normais primitivos: $v_{1}=(-1,0), v_{2}=(0,-1), v_{3}=(1,1)$

$$
\begin{aligned}
\Delta & =\left\{x=\left(x_{1}, x_{2}\right) \in\left(\mathbb{R}^{2}\right)^{*} \mid x_{1} \geq 0, x_{2} \geq 0, x_{1}+x_{2} \leq L\right\} \\
& =\left\{x \in\left(\mathbb{R}^{2}\right)^{*} \mid\langle x,(-1,0)\rangle \leq 0,\langle x,(0,-1)\rangle \leq 0,\langle x,(1,1)\rangle \leq L\right\} \\
\Rightarrow \lambda_{1} & =\lambda_{2}=0, \lambda_{3}=L .
\end{aligned}
$$

Passo 2 Determinar o núcleo $N$ e a aplicação inclusão $i$

- Projeção $\pi$ :

$$
\begin{aligned}
\pi: \mathbb{R}^{3} & \longrightarrow \mathbb{R}^{2} \\
e_{1} & \longmapsto v_{1} \\
e_{2} & \longmapsto v_{2} \\
e_{3} & \longmapsto v_{3}
\end{aligned}
$$

Assim

$$
\begin{aligned}
& \pi(1,0,0)=(-1,0) \\
& \pi(0,1,0)=(0,-1) \\
& \pi(0,0,1)=(1,1)
\end{aligned} \Rightarrow \pi_{2 \times 3}=\left[\begin{array}{rrr}
-1 & 0 & 1 \\
0 & -1 & 1
\end{array}\right]
$$


. Toro $N$ :

$$
\begin{aligned}
N=\operatorname{ker} \pi \Rightarrow(a, b, c) \in N & \Leftrightarrow\left[\begin{array}{rrr}
-1 & 0 & 1 \\
0 & -1 & 1
\end{array}\right]\left[\begin{array}{l}
a \\
b \\
c
\end{array}\right]=\left[\begin{array}{l}
0 \\
0
\end{array}\right] \\
& \Leftrightarrow-a+c=0,-b+c=0 \\
& \Leftrightarrow(a, b, c)=(a, a, a)=a(1,1,1) .
\end{aligned}
$$

Disto, segue que $\operatorname{dim}(N)=1 \Rightarrow N=\mathbb{S}^{1}$ e, em particular, $N$ é o $\mathbb{S}^{1}$ diagonal em $\mathbb{T}^{3}$. Temos, portanto, a sequência exata de toros e de suas respectivas álgebras de Lie:

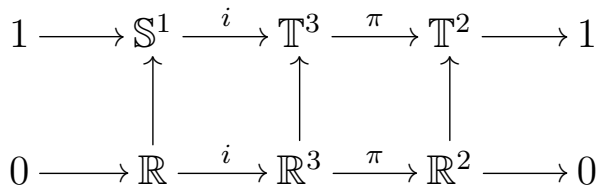

- Aplicação inclusão:

$$
\begin{aligned}
i: \mathbb{R} & \longrightarrow \mathbb{R}^{3} \\
a & \longmapsto(a, a, a)=a(1,1,1)
\end{aligned} \Rightarrow i_{3 \times 1}=\left[\begin{array}{lll}
1 & 1 & 1
\end{array}\right]^{T}
$$

- Dual da aplicação inclusão: Temos abaixo a sequência exata do dual das álgebras de Lie

$$
0 \longrightarrow\left(\mathbb{R}^{2}\right)^{*}=\mathbb{R}^{2} \stackrel{\pi^{*}}{\longrightarrow}\left(\mathbb{R}^{3}\right)^{*}=\mathbb{R}^{3} \stackrel{i^{*}}{\longrightarrow} \mathbb{R}^{*}=\mathbb{R} \longrightarrow 0
$$

Como $i^{*}=[i]^{T}=\left[\begin{array}{lll}1 & 1 & 1\end{array}\right]$, então

$$
\begin{aligned}
i^{*}: \mathbb{R}^{3} & \longrightarrow \mathbb{R} \\
(a, b, c) & \longmapsto a+b+c
\end{aligned}
$$

Passo 3 Determinar a aplicação momento $\mu$, o conjunto de nível $Z$ e a variedade reduzida $M_{\Delta}$.

- Aplicação momento: Consideremos a ação diagonal de $\mathbb{T}^{3}$ sobre a variedade $\left(\mathbb{C}^{3}, \omega_{s t d}=\frac{i}{2} \sum_{i=1}^{3} d z_{i} \wedge d \bar{z}_{i}\right)$ com aplicação momento

$$
\begin{aligned}
& \mu: \quad \mathbb{C}^{3} \longrightarrow \mathbb{R}^{3} \\
&\left(z_{1}, z_{2}, z_{3}\right) \longmapsto-\frac{1}{2}\left(\left|z_{1}\right|^{2},\left|z_{2}\right|^{2},\left|z_{3}\right|^{2}\right)+\left(\lambda_{1}, \lambda_{2}, \lambda_{3}\right) \\
& \Rightarrow \mu\left(z_{1}, z_{2}, z_{3}\right)=\left(-\frac{\left|z_{1}\right|^{2}}{2},-\frac{\left|z_{2}\right|^{2}}{2}, L-\frac{\left|z_{3}\right|^{2}}{2}\right) .
\end{aligned}
$$


Além disso, temos $\mu_{N}=i^{*} \circ \mu: \mathbb{C}^{3} \longrightarrow \mathbb{R}$ tal que

$$
\begin{aligned}
\mu_{N}\left(z_{1}, z_{2}, z_{3}\right) & =i^{*} \circ \mu\left(z_{1}, z_{2}, z_{3}\right) \\
& =i^{*}\left(-\frac{\left|z_{1}\right|^{2}}{2},-\frac{\left|z_{2}\right|^{2}}{2}, L-\frac{\left|z_{3}\right|^{2}}{2}\right) \\
& =-\frac{1}{2}\left(\left|z_{1}\right|^{2}+\left|z_{2}\right|^{2}+\left|z_{3}\right|^{2}\right)+L .
\end{aligned}
$$

- Conjunto de nível zero:

$Z=\mu_{N}^{-1}(0)$, então

$-\frac{1}{2}\left(\left|z_{1}\right|^{2}+\left|z_{2}\right|^{2}+\left|z_{3}\right|^{2}\right)+L=0 \Leftrightarrow L=\frac{1}{2}\left(\left|z_{1}\right|^{2}+\left|z_{2}\right|^{2}+\left|z_{3}\right|^{2}\right) \subset \mathbb{C}^{3} \simeq \mathbb{R}^{6}$.

Logo, $Z=\mathbb{S}_{\sqrt{2 L}}^{5}$ (observe aqui que $\operatorname{dim}_{\mathbb{R}} Z=d+n=5$ ).

- Variedade reduzida:

$$
M_{\Delta}=\frac{Z}{N}=\frac{\mathbb{S}_{\sqrt{2 L}}^{5}}{\mathbb{S}^{1}} \simeq \mathbb{C P}^{2}
$$

Pelo Teorema 6.7, existe uma estrutura simplética sobre $\mathbb{C P}^{2}$. Como no caso da linha projetiva, a 2-forma sobre $\mathbb{C P}^{2}$ obtida pela redução simplética se chama de forma Fubini-Study.

\section{3}

Quadrado de Lado $L$

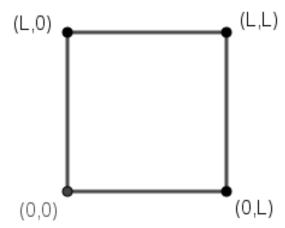

Passo 1 Descrição do polítopo

- Dimensão: $n=2$.

- Facetas: $d=4$. 


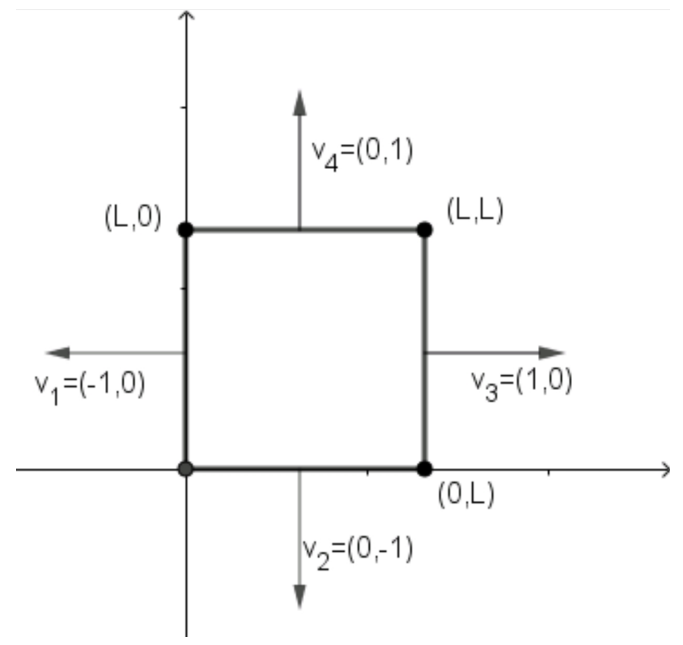

- Vetores normais primitivos: $v_{1}=(-1,0), v_{2}=(0,-1), v_{3}=(1,0) \mathrm{e}$ $v_{4}=(0,1)$.

$$
\begin{aligned}
\Delta & =\left\{x=\left(x_{1}, x_{2}\right) \in\left(\mathbb{R}^{2}\right)^{*} \mid 0 \leq x_{1} \leq L, 0 \leq x_{2} \leq L\right\} \\
& =\left\{x \in\left(\mathbb{R}^{2}\right)^{*} \mid\langle x,(-1,0)\rangle \leq 0,\langle x,(0,-1)\rangle \leq 0,\langle x,(1,0)\rangle \leq L,\langle x,(0,1)\rangle \leq L\right\} \\
\Rightarrow & \lambda_{1}=\lambda_{2}=0, \lambda_{3}=\lambda_{4}=L .
\end{aligned}
$$

Passo 2 Determinar o núcleo $N$ e a aplicação inclusão $i$

- Projeção $\pi$ :

$$
\begin{aligned}
\pi: \mathbb{R}^{4} & \longrightarrow \mathbb{R}^{2} \\
e_{1} & \longmapsto v_{1} \\
e_{2} & \longmapsto v_{2} \\
e_{3} & \longmapsto v_{3} \\
e_{4} & \longmapsto v_{4}
\end{aligned}
$$

\section{Assim}

$$
\begin{aligned}
& \pi(1,0,0,0)=(-1,0) \\
& \pi(0,1,0,0)=(0,-1) \\
& \pi(0,0,1,0)=(1,0) \\
& \pi(0,0,1,0)=(0,1)
\end{aligned} \quad \Rightarrow \pi_{2 \times 4}=\left[\begin{array}{rrrr}
-1 & 0 & 1 & 0 \\
0 & -1 & 0 & 1
\end{array}\right]
$$


- Toro $N$ :

$$
\begin{aligned}
N=\operatorname{ker} \pi \Rightarrow(a, b, c, d) \in N & \Leftrightarrow\left[\begin{array}{rrrr}
-1 & 0 & 1 & 0 \\
0 & -1 & 0 & 1
\end{array}\right]\left[\begin{array}{l}
a \\
b \\
c \\
d
\end{array}\right]=\left[\begin{array}{l}
0 \\
0
\end{array}\right] \\
& \Leftrightarrow-a+c=0,-b+d=0 \\
& \Leftrightarrow(a, b, c, d)=(a, b, a, b) .
\end{aligned}
$$

Disto, segue que $\operatorname{dim}(N)=2 \Rightarrow N=\mathbb{T}^{2}=\mathbb{S}^{1} \times \mathbb{S}^{1}$. Temos, portanto, a sequência exata de toros e de suas respectivas álgebras de Lie:

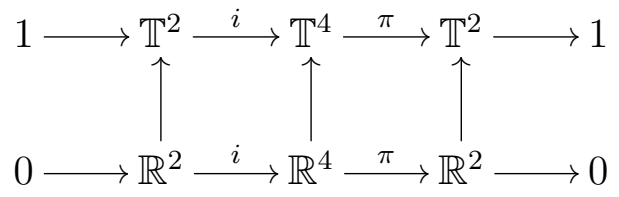

- Aplicação inclusão:

$$
\begin{aligned}
i: \mathbb{R}^{2} & \longrightarrow \mathbb{R}^{4} \\
(a, b) & \longmapsto(a, b, a, b)=a(1,0,1,0)+b(0,1,0,1)
\end{aligned} \Rightarrow i_{4 \times 2}=\left[\begin{array}{ll}
1 & 0 \\
0 & 1 \\
1 & 0 \\
0 & 1
\end{array}\right]
$$

- Dual da aplicação inclusão: Temos abaixo a sequência exata do dual das álgebras de Lie

$$
\begin{aligned}
0 \longrightarrow & \left(\mathbb{R}^{2}\right)^{*}=\mathbb{R}^{2} \stackrel{\pi^{*}}{\longrightarrow}\left(\mathbb{R}^{4}\right)^{*}=\mathbb{R}^{4} \stackrel{i^{*}}{\longrightarrow}\left(\mathbb{R}^{2}\right)^{*}=\mathbb{R}^{2} \longrightarrow 0 \\
\text { Como } i^{*}=[i]^{T}=\left[\begin{array}{llll}
1 & 0 & 1 & 0 \\
0 & 1 & 0 & 1
\end{array}\right] \text {, então } & \\
i^{*}: \quad \mathbb{R}^{4} \stackrel{\mathbb{R}^{2}}{ } & \longrightarrow(a+c, b+d) .
\end{aligned}
$$

Passo 3 Determinar a aplicação momento $\mu$, o conjunto de nível $Z$ e a variedade reduzida $M_{\Delta}$.

- Aplicação momento da ação do grupo sobre a variedade:

$$
\begin{aligned}
\mu: \mathbb{C}^{4} & \longrightarrow \mathbb{R}^{4} \\
\left(z_{1}, z_{2}, z_{3}, z_{4}\right) & \longmapsto-\frac{1}{2}\left(\left|z_{1}\right|^{2},\left|z_{2}\right|^{2},\left|z_{3}\right|^{2},\left|z_{4}\right|^{2}\right)+\left(\lambda_{1}, \lambda_{2}, \lambda_{3}, \lambda_{4}\right)
\end{aligned}
$$




$$
\Rightarrow \mu\left(z_{1}, z_{2}, z_{3}, z_{4}\right)=\left(-\frac{\left|z_{1}\right|^{2}}{2},-\frac{\left|z_{2}\right|^{2}}{2}, L-\frac{\left|z_{3}\right|^{2}}{2}, L-\frac{\left|z_{4}\right|^{2}}{2}\right) .
$$

Além disso, temos $\mu_{N}=i^{*} \circ \mu: \mathbb{C}^{4} \longrightarrow \mathbb{R}^{2}$ tal que

$$
\begin{aligned}
\mu_{N}\left(z_{1}, z_{2}, z_{3}, z_{4}\right) & =i^{*} \circ \mu\left(z_{1}, z_{2}, z_{3}, z_{4}\right) \\
& =i^{*}\left(-\frac{\left|z_{1}\right|^{2}}{2},-\frac{\left|z_{2}\right|^{2}}{2}, L-\frac{\left|z_{3}\right|^{2}}{2}, L-\frac{\left|z_{4}\right|^{2}}{2}\right) \\
& =\left(L-\frac{\left|z_{1}\right|^{2}+\left|z_{3}\right|^{2}}{2}, L-\frac{\left|z_{2}\right|^{2}+\left|z_{4}\right|^{2}}{2}\right)
\end{aligned}
$$

- Conjunto de nível zero:

$Z=\mu_{N}^{-1}(0)=\left\{\left.\left(z_{1}, z_{2}, z_{3}, z_{4}\right) \in \mathbb{C}^{4}|| z_{1}\right|^{2}+\left|z_{3}\right|^{2}=2 L,\left|z_{2}\right|^{2}+\left|z_{4}\right|^{2}=2 L\right\}$

Logo, $Z=\mathbb{S}_{\sqrt{2 L}}^{3} \times \mathbb{S}_{\sqrt{2 L}}^{3}$ (observe aqui que $\operatorname{dim}_{\mathbb{R}} Z=d+n=6$ ).

- Variedade reduzida:

$$
M_{\Delta}=\frac{Z}{N}=\frac{\mathbb{S}_{\sqrt{2 L}}^{3} \times \mathbb{S}_{\sqrt{2 L}}^{3}}{\mathbb{T}^{2}}=\frac{\mathbb{S}_{\sqrt{2 L}}^{3} \times \mathbb{S}_{\sqrt{2 L}}^{3}}{\mathbb{S}^{1} \times \mathbb{S}^{1}} \simeq \mathbb{C P}^{1} \times \mathbb{C P}^{1}
$$

Como calculada anteriormente, a ação de $N$ em um ponto $\left(z_{1}, z_{2}, z_{3}, z_{4}\right) \in Z$ é $(a, b, a, b) \cdot\left(z_{1}, z_{2}, z_{3}, z_{4}\right)=\left(a z_{1}, b z_{2}, a z_{3}, b z_{4}\right)$ e a estrutura simplética obtida por redução sobre $\mathbb{C P}^{1} \times \mathbb{C P}^{1}$ é $\omega_{\text {red }}=\sum_{i=1}^{2} \pi_{i}^{*}\left(\omega_{F S}\right)$ onde temos $\pi_{i}: \mathbb{C P}^{1} \times \mathbb{C P}^{1} \longrightarrow \mathbb{C P}^{1}$ é a projeção sobre o $i$-ésimo fator.

\section{4}

\section{Trapézio}

Passo 1 Descrição do polítopo

- Dimensão: $n=2$.

- Facetas: $d=4$.

- Vetores normais primitivos: $v_{1}=(-1,0), v_{2}=(0,-1), v_{3}=(1, L) \mathrm{e}$ $v_{4}=(0,1)$.

$$
\begin{aligned}
& \Delta=\left\{x=\left(x_{1}, x_{2}\right) \in\left(\mathbb{R}^{2}\right)^{*} \mid 0 \leq x_{1} \leq L+1,0 \leq x_{2} \leq 1, x_{1}+L x_{2} \leq L+1\right\} \\
& \quad=\left\{x \in\left(\mathbb{R}^{2}\right)^{*} \mid\langle x,(-1,0)\rangle \leq 0,\langle x,(0,-1)\rangle \leq 0,\langle x,(1, L)\rangle \leq L+1,\langle x,(0,1)\rangle \leq 1\right\} \\
& \Rightarrow \lambda_{1}=\lambda_{2}=0, \lambda_{3}=L+1, \lambda_{4}=1
\end{aligned}
$$




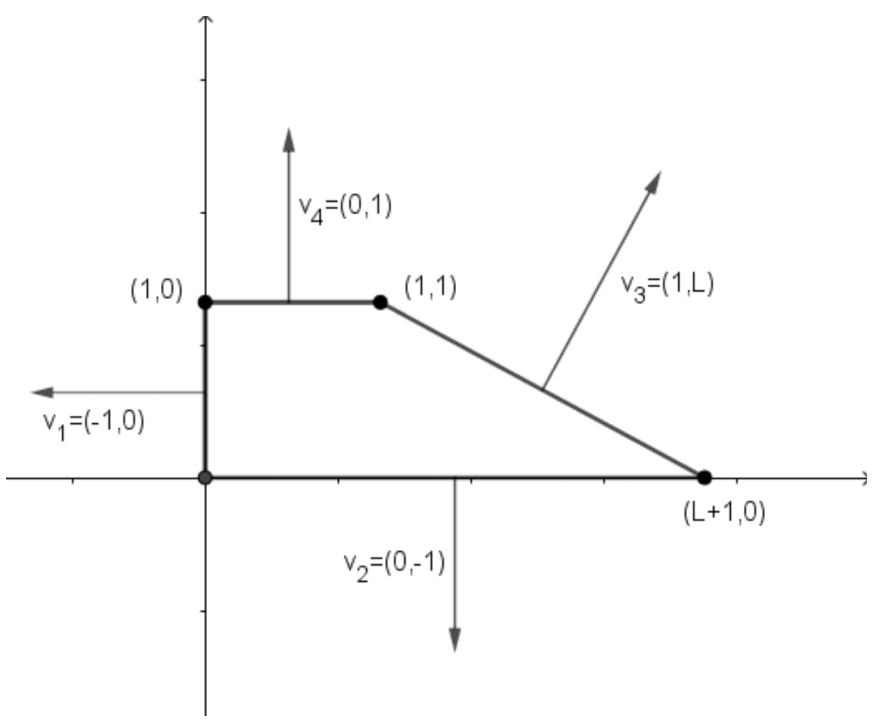

Passo 2: Determinar o núcleo $N$ e a aplicação inclusão $i$

. Projeção $\pi$ :

$$
\begin{aligned}
\pi: \mathbb{R}^{4} & \longrightarrow \mathbb{R}^{2} \\
e_{1} & \longmapsto v_{1} \\
e_{2} & \longmapsto v_{2} \\
e_{3} & \longmapsto v_{3} \\
e_{4} & \longmapsto v_{4}
\end{aligned}
$$

\section{Assim}

$$
\begin{aligned}
& \pi(1,0,0,0)=(-1,0) \\
& \pi(0,1,0,0)=(0,-1) \\
& \pi(0,0,1,0)=(1, L) \\
& \pi(0,0,1,0)=(0,1)
\end{aligned} \quad \Rightarrow \pi_{2 \times 4}=\left[\begin{array}{rrrr}
-1 & 0 & 1 & 0 \\
0 & -1 & L & 1
\end{array}\right]
$$

- Toro $N$ :

$$
\begin{aligned}
& N=\operatorname{ker} \pi \Rightarrow(a, b, c, d) \in N \Leftrightarrow\left[\begin{array}{rrrr}
-1 & 0 & 1 & 0 \\
0 & -1 & L & 1
\end{array}\right]\left[\begin{array}{l}
a \\
b \\
c \\
d
\end{array}\right]=\left[\begin{array}{l}
0 \\
0
\end{array}\right] \\
& \Leftrightarrow\left\{\begin{array}{l}
-a+c=0 \\
-b+L c+d=0
\end{array}\right. \\
& \Leftrightarrow\left\{\begin{array}{l}
a=c \\
d=b-L a
\end{array}\right.
\end{aligned}
$$


Disto, segue que $\operatorname{dim}(N)=2 \Rightarrow N=\mathbb{T}^{2}=\mathbb{S}^{1} \times \mathbb{S}^{1}$. Temos, portanto, a sequência exata de toros e de suas respectivas álgebras de Lie:

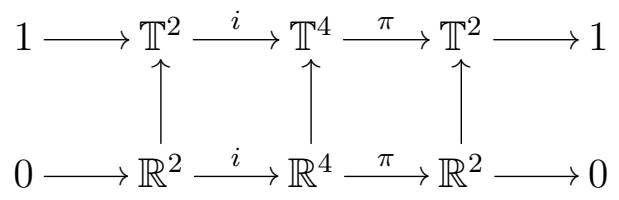

- Aplicação inclusão:

$$
\begin{aligned}
& i: \quad \mathbb{R}^{2} \longrightarrow \mathbb{R}^{4} \\
& (a, b) \longmapsto(a, b, a, b-L a)=a(1,0,1,-L)+b(0,1,0,1) \\
& \Rightarrow i_{4 \times 2}=\left[\begin{array}{cc}
1 & 0 \\
0 & 1 \\
1 & 0 \\
-L & 1
\end{array}\right]
\end{aligned}
$$

- Dual da aplicação inclusão: Temos abaixo a sequência exata do dual das álgebras de Lie

$$
\begin{aligned}
& 0 \longrightarrow\left(\mathbb{R}^{2}\right)^{*}=\mathbb{R}^{2} \stackrel{\pi^{*}}{\longrightarrow}\left(\mathbb{R}^{4}\right)^{*}=\mathbb{R}^{4} \stackrel{i^{*}}{\longrightarrow}\left(\mathbb{R}^{2}\right)^{*}=\mathbb{R}^{2} \longrightarrow 0 \\
& \text { Como } i^{*}=[i]^{T}=\left[\begin{array}{cccc}
1 & 0 & 1 & -L \\
0 & 1 & 0 & 1
\end{array}\right] \text {, então } \\
& i^{*}: \quad \mathbb{R}^{4} \longrightarrow \mathbb{R}^{2} \\
& (a, b, c, d) \longmapsto(a+c-L d, b+d) \text {. }
\end{aligned}
$$

Passo 3 Determinar a aplicação momento $\mu$, o conjunto de nível $Z$ e a variedade reduzida $M_{\Delta}$.

- Aplicação momento: Consideremos a ação diagonal de $\mathbb{T}^{4}$ sobre a variedade $\left(\mathbb{C}^{4}, \omega_{s t d}=\frac{i}{2} \sum_{i=1}^{4} d z_{i} \wedge d \bar{z}_{i}\right)$ com aplicação momento

$$
\begin{aligned}
& \mu: \quad \mathbb{C}^{4} \longrightarrow \mathbb{R}^{4} \\
&\left(z_{1}, z_{2}, z_{3}, z_{4}\right) \longmapsto-\frac{1}{2}\left(\left|z_{1}\right|^{2},\left|z_{2}\right|^{2},\left|z_{3}\right|^{2},\left|z_{4}\right|^{2}\right)+\left(\lambda_{1}, \lambda_{2}, \lambda_{3}, \lambda_{4}\right) \\
& \Rightarrow \mu\left(z_{1}, z_{2}, z_{3}, z_{4}\right)=\left(-\frac{\left|z_{1}\right|^{2}}{2},-\frac{\left|z_{2}\right|^{2}}{2}, L+1-\frac{\left|z_{3}\right|^{2}}{2}, 1-\frac{\left|z_{4}\right|^{2}}{2}\right) .
\end{aligned}
$$


Além disso, temos $\mu_{N}=i^{*} \circ \mu: \mathbb{C}^{4} \longrightarrow \mathbb{R}^{2}$ tal que

$$
\begin{aligned}
\mu_{N}\left(z_{1}, z_{2}, z_{3}, z_{4}\right) & =i^{*} \circ \mu\left(z_{1}, z_{2}, z_{3}, z_{4}\right) \\
& =i^{*}\left(-\frac{\left|z_{1}\right|^{2}}{2},-\frac{\left|z_{2}\right|^{2}}{2}, L+1-\frac{\left|z_{3}\right|^{2}}{2}, 1-\frac{\left|z_{4}\right|^{2}}{2}\right) \\
& =\left(1-\frac{\left|z_{1}\right|^{2}+\left|z_{3}\right|^{2}-L\left|z_{4}\right|^{2}}{2}, 1-\frac{\left|z_{2}\right|^{2}+\left|z_{4}\right|^{2}}{2}\right)
\end{aligned}
$$

- Conjunto de nível zero:

$Z=\mu_{N}^{-1}(0)=\left\{\left.\left(z_{1}, z_{2}, z_{3}, z_{4}\right) \in \mathbb{C}^{4}|| z_{1}\right|^{2}+\left|z_{3}\right|^{2}-L\left|z_{4}\right|^{2}=2,\left|z_{2}\right|^{2}+\right.$ $\left.\left|z_{4}\right|^{2}=2\right\}$.

Sabemos que a segunda equação se trata da esfera $\mathbb{S}_{\sqrt{2}}^{3}$ e que a dimensão real de $Z$ é calculada por $\operatorname{dim}_{\mathbb{R}} Z=d+n=6$, mas resta estudar com cuidado a primeira equação.

- Variedade reduzida:

Lembremos que a ação do toro $N$ sobre um ponto $\left(z_{1}, z_{2}, z_{3}, z_{4}\right) \in Z$ é

$$
(a, b, a, b-L a) \cdot\left(z_{1}, z_{2}, z_{3}, z_{4}\right)=\left(a z_{1}, b z_{2}, a z_{3},(b-L a) z_{4}\right) .
$$

O associado espaço das órbitas, i.e, a variedade reduzida é

$$
M_{\Delta}=\frac{Z}{N}=\mathbb{S}_{\sqrt{2}}^{3} \times_{\mathbb{S}^{1}} \mathbb{C P}^{1}
$$

onde, usando em coordenadas $\left(\left(z_{1}, z_{3}\right),\left[z_{2}: z_{4}\right]\right)$ em $\mathbb{S}_{\sqrt{2}}^{3} \times \mathbb{C P}^{1}$ com a relação $\left|z_{1}\right|^{2}+\left|z_{3}\right|^{2}-L\left|z_{4}\right|^{2}=2$, temos que a ação de $\mathbb{S}^{1}$ é

$$
\begin{aligned}
a \cdot\left(\left(z_{1}, z_{3}\right),\left[z_{2}: z_{4}\right]\right)= & \begin{array}{c}
\left(\left(a z_{1}, a z_{3}\right),\left[z_{2}:-L a z_{4}\right]\right) \\
\| \\
\left(\left(a z_{1}, a z_{3}\right),\left[-(L a)^{-1} z_{2}: z_{4}\right]\right) .
\end{array}
\end{aligned}
$$

Segue que a variedade simplética reduzida $M_{\Delta}$ é uma fibração sobre $\mathbb{C P}^{1}$ com fibra $\mathbb{C P}^{1}$, chamada Superfície de Hirzebruch. Para aprofundar, consulte [17] e [16]. 


\section{5}

\section{Tetraedro}

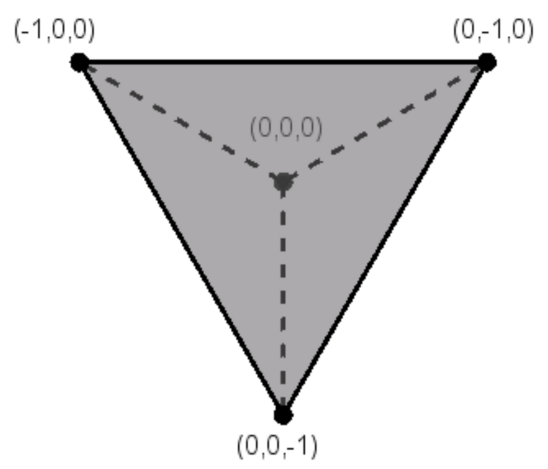

Passo 1 Descrição do polítopo

- Dimensão: $n=3$.

- Facetas: $d=4$.

- Vetores normais primitivos:

$$
\begin{aligned}
& v_{1}=(1,0,0), v_{2}=(0,1,0), v_{3}=(0,0,1), v_{4}=(-1,-1,-1) \\
& \begin{aligned}
& \Delta=\left\{x=\left(x_{1}, x_{2}, x_{3}\right) \in\left(\mathbb{R}^{3}\right)^{*} \mid x_{1} \leq 0, x_{2} \leq 0, x_{3} \leq 0, x_{1}+x_{2}+x_{3} \geq 1\right\} \\
&=\left\{x \in\left(\mathbb{R}^{3}\right)^{*} \mid\right.\langle x,(1,0,0)\rangle \leq 0, \quad\langle x,(0,1,0)\rangle \leq 0, \\
&\langle x,(0,0,1)\rangle \leq 0, \quad\langle x,(-1,-1,-1)\rangle \leq 1\}
\end{aligned} \\
& \Rightarrow \lambda_{1}=\lambda_{2}=\lambda_{3}=0, \lambda_{4}=1 .
\end{aligned}
$$

Passo 2 Determinar o núcleo $N$ e a aplicação inclusão $i$

. Projeção $\pi$ :

temos

$$
\begin{aligned}
& \pi(1,0,0,0)=(1,0,0) \\
& \pi(0,1,0,0)=(0,1,0) \\
& \pi(0,0,1,0)=(0,0,1) \\
& \pi(0,0,1,0)=(-1,-1,-1)
\end{aligned} \quad \Rightarrow \pi_{3 \times 4}=\left[\begin{array}{cccc}
1 & 0 & 0 & -1 \\
0 & 1 & 0 & -1 \\
0 & 0 & 1 & -1
\end{array}\right]
$$


- Toro $N$ :

$$
\begin{aligned}
& N=\operatorname{ker} \pi \Rightarrow(a, b, c, d) \in N \Leftrightarrow\left[\begin{array}{cccc}
1 & 0 & 0 & -1 \\
0 & 1 & 0 & -1 \\
0 & 0 & 1 & -1
\end{array}\right]\left[\begin{array}{l}
a \\
b \\
c \\
d
\end{array}\right]=\left[\begin{array}{l}
0 \\
0 \\
0
\end{array}\right] \\
& \Leftrightarrow\left\{\begin{array}{l}
a-d=0 \\
b-d=0 \\
c-d=0
\end{array}\right. \\
& \Leftrightarrow a=b=c=d .
\end{aligned}
$$

Disto, segue que $\operatorname{dim}(N)=1 \Rightarrow N=\mathbb{S}^{1}$. Temos, portanto, a sequência exata de toros e de suas respectivas álgebras de Lie:

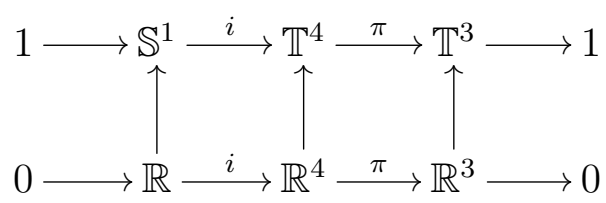

- Aplicação inclusão:

$$
\begin{aligned}
i: \mathbb{R} & \longrightarrow \mathbb{R}^{4} \\
a & \longmapsto(a, a, a, a)=a(1,1,1,1)
\end{aligned} \Rightarrow i_{4 \times 1}=\left[\begin{array}{l}
1 \\
1 \\
1 \\
1
\end{array}\right]
$$

- Dual da aplicação inclusão: Temos abaixo a sequência exata do dual das álgebras de Lie

$$
0 \longrightarrow\left(\mathbb{R}^{3}\right)^{*}=\mathbb{R}^{3} \stackrel{\pi^{*}}{\longrightarrow}\left(\mathbb{R}^{4}\right)^{*}=\mathbb{R}^{4} \stackrel{i^{*}}{\longrightarrow} \mathbb{R}^{*}=\mathbb{R} \longrightarrow 0
$$

$$
\begin{aligned}
\text { Como } i^{*}=[i]^{T}=\left[\begin{array}{llll}
1 & 1 & 1 & 1
\end{array}\right] \text {, então } & \\
& \begin{aligned}
i^{*}: & \mathbb{R}^{4} \\
(a, b, c, d) & \longmapsto \mathbb{R} \\
& \longmapsto a+b+c+d .
\end{aligned}
\end{aligned}
$$

Passo 3 Determinar a aplicação momento $\mu$, o conjunto de nível $Z$ e a variedade reduzida $M_{\Delta}$. 
- Aplicação momento da ação do grupo sobre a variedade:

$$
\begin{aligned}
& \mu: \quad \mathbb{C}^{4} \longrightarrow \mathbb{R}^{4} \\
&\left(z_{1}, z_{2}, z_{3}, z_{4}\right) \longmapsto-\frac{1}{2}\left(\left|z_{1}\right|^{2},\left|z_{2}\right|^{2},\left|z_{3}\right|^{2},\left|z_{4}\right|^{2}\right)+\left(\lambda_{1}, \lambda_{2}, \lambda_{3}, \lambda_{4}\right) \\
& \Rightarrow \mu\left(z_{1}, z_{2}, z_{3}, z_{4}\right)=\left(-\frac{\left|z_{1}\right|^{2}}{2},-\frac{\left|z_{2}\right|^{2}}{2},-\frac{\left|z_{3}\right|^{2}}{2}, 1-\frac{\left|z_{4}\right|^{2}}{2}\right) .
\end{aligned}
$$

Além disso, temos $\mu_{N}=i^{*} \circ \mu: \mathbb{C}^{4} \longrightarrow \mathbb{R}$ tal que

$$
\begin{aligned}
\mu_{N}\left(z_{1}, z_{2}, z_{3}, z_{4}\right) & =i^{*} \circ \mu\left(z_{1}, z_{2}, z_{3}, z_{4}\right) \\
& =i^{*}\left(-\frac{\left|z_{1}\right|^{2}}{2},-\frac{\left|z_{2}\right|^{2}}{2},-\frac{\left|z_{3}\right|^{2}}{2}, 1-\frac{\left|z_{4}\right|^{2}}{2}\right) \\
& =1-\frac{\left|z_{1}\right|^{2}+\left|z_{2}\right|^{2}+\left|z_{3}\right|^{2}+\left|z_{4}\right|^{2}}{2}
\end{aligned}
$$

- Conjunto de nível zero:

$Z=\mu_{N}^{-1}(0)=\left\{\left.\left(z_{1}, z_{2}, z_{3}, z_{4}\right) \in \mathbb{C}^{4}|| z_{1}\right|^{2}+\left|z_{2}\right|^{2}+\left|z_{3}\right|^{2}+\left|z_{4}\right|=2\right\}=\mathbb{S}_{\sqrt{2}}^{7}$

- Variedade reduzida:

$$
M_{\Delta}=\frac{Z}{N}=\frac{\mathbb{S}_{\sqrt{2}}^{7}}{\mathbb{S}^{1}}=\mathbb{C P}^{3}
$$

Análogo às seções 8.1 e 8.2, pelo Teorema 6.7, existe uma estrutura simplética sobre $\mathbb{C P}^{3}$ chamada de forma Fubini-Study.

8.6

\section{Cubo de Lado $L$}

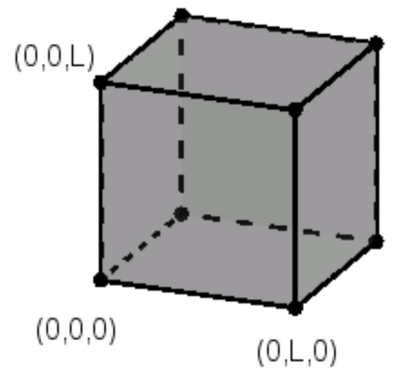


Este exemplo está diretamente ligado aos exemplos 8.1 e 8.3. De fato, temos que, dadas duas variedades tóricas simpléticas $2 n$-dimensionais $\left(M, \omega_{M}, \mathbb{T}^{n}, \mu_{M}\right)$ e $\left(N, \omega_{N}, \mathbb{T}^{n}, \mu_{N}\right)$, a variedade tórica simplética produto é descrita por

$$
\left(M \times N, \omega=\pi_{1}^{*} \omega_{M}+\pi_{2}^{*} \omega_{N}, \mathbb{T}^{n}, \mu=\mu_{M}+\mu_{N}\right),
$$

com o grupo $\mathbb{T}^{n}$ atuando diagonalmente sobre $M$ e $N$ e $\pi_{i}$ como a projeção de $M \times N$ sobre o primeiro e segundo fatores, respectivamente, para $i=\{1,2\}$. Então

$$
\left(\mu_{M}+\mu_{N}\right)(M \times N)=\mu_{M}(M) \times \mu_{N}(N) .
$$

Prova. Suponha $\left(p_{1}, p_{2}\right) \in\left(\mu_{M}+\mu_{N}\right)(M \times N)$. Assim, existe $(m, n) \in M \times N$ tal que

$$
\left(\mu_{M}+\mu_{N}\right)(m, n)=\left(p_{1}, p_{2}\right) \Leftrightarrow\left(\mu_{M}(m), \mu_{N}(n)\right)=\left(p_{1}, p_{2}\right) .
$$

Disto, temos a variedade reduzida com dimensão real igual a 6 , isto é, igual ao dobro da dimensão do polítopo cubo de lado $L$. Portanto,

$$
M_{\Delta}=\mathbb{C P}^{1} \times \mathbb{C P}^{1} \times \mathbb{C P}^{1}
$$

com estrutura simplética $\omega_{\text {red }}=\sum_{i=1}^{3} \pi_{i}^{*}\left(\omega_{F S}\right)$, para $\pi_{i}: \mathbb{C P}^{1} \times \mathbb{C P}^{1} \times \mathbb{C P}^{1} \longrightarrow \mathbb{C P}^{1}$ sendo a projeção sobre o $i$-ésimo fator. 


\section{Referências bibliográficas}

[1] DELZANT, T. Hamiltoniens périodiques et images convexes de l'application moment. Bull. Soc. Math. France, 116(3):315-339, 1988.

[2] MARSDEN, J., WEINSTEIN, A., Reduction of symplectic manifolds with symmetry, Rep. Mathematical Phys. 5 (1974), 121-130.

[3] MEYER, K., Symmetries and integrals in mechanics, Dynamical Systems Proc. Sympos., Univ. Bahia, Salvador, 1971, 259-272. Academic Press, New York, 1973.

[4] DO CARMO , M. Differential Forms and Applications, Springer, Berlin, 1994.

[5] FERNANDES, R. L. Lições de Geometria Diferencial, notas de aula. Departamento de Matemática, IST. Lisboa, 2003.

[6] LEE, J. M. Introduction to Smooth Manifolds, Graduate Texts in Mathematics, Vol. 218, Springer, New York, 2003.

[7] MCDUFF, D., SALAMON, D. What is Symplectic Geometry? Talk given at the European Women in Mathematics conference of Sept 2007 in Cambridge, UK, 2009.

[8] BERNDT, R. An introduction to symplectic geometry, AMS Graduate Studies in mathematics 26, 2001.

[9] TU, L. W. An Introduction to Manifolds, Springer, 2008.

[10] LIMA, E. L. Variedades Diferenciáveis. Publicações Matemáticas. Rio de Janeiro: IMPA, 2010.

[11] BURSZTYN, H.; MACARINI, L. Introdução a Geometria Simplética.Rio de Janeiro: IMPA, XIV Escola de Geometria Diferencial, 2006.

[12] ARNOLD, V., Mathematical Methods of Classical Mechanics, Graduate Texts in Mathematics 60, Springer-Verlag, New York, 1978.

[13] ATIYAH, M., Convexity and commuting Hamiltonians, Bull. London Math. Soc. 14 (1982), 1-15. 
[14] GUILLEMIN, V., STERNBERG, S.,Convexity properties of the moment mapping, Invent. Math. 67 (1982), 491-513.

[15] CANNAS, A. Lectures on Symplectic Geometry, Lectures Notes in Math. 1764, Springer-Verlag, 2001.

[16] MCDUFF, D., SALAMON, D. Introduction to Symplectic Topology, second edition, Oxford Mathematical Monographs, Oxford University Press, 1998.

[17] AUDIN, M. Torus Actions on Symplectic Manifolds, second edition, Progress in Mathematics 93, Birkhauser Verlag, Basel, 2004. 